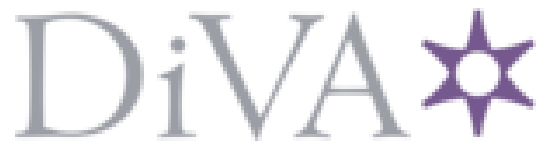

http://www.diva-portal.org

\title{
Postprint
}

This is the accepted version of a paper published in Nonlinear dynamics. This paper has been peer-reviewed but does not include the final publisher proof-corrections or journal pagination.

Citation for the original published paper (version of record):

Sun, D., Naghdy, F., Du, H. (2017)

Time domain passivity control of time-delayed bilateral telerobotics with prescribed performance.

Nonlinear dynamics, 87: 1253-1270

https://doi.org/10.1007/s11071-016-3113-6

Access to the published version may require subscription.

N.B. When citing this work, cite the original published paper.

Permanent link to this version:

http://urn.kb.se/resolve?urn=urn:nbn:se:oru:diva-64479 


\section{Time Domain Passivity Control of Time Delayed Bilateral Telerobotics with Prescribed Performance}

\begin{abstract}
A novel approach applying the extended Prescribed Performance Control (PPC) and the wave-based Time Domain Passivity Approach (wave-based TDPA) to teleoperation systems is proposed. With the extended PPC, a teleoperation system can synchronize position, velocity and force. Moreover, by combining with the extended wave-based TDPA, the overall system's passivity is guaranteed in the presence of arbitrary time delays. The system's stability and performance are analyzed by using Lyapunov functions. The method is validated through experimental work based on a 3DOF bilateral teleoperation system. The experimental results show that the proposed control algorithm can robustly guarantee the master-slave system's passivity and simultaneously provide high tracking performance of position, velocity and measured force signals.
\end{abstract}

Da Sun, Fazel Naghdy and Haiping Du

Index Terms - Bilateral teleoperation, Prescribed Performance Control (PPC) Time Domain Passivity Approach (TDPA), Wave variable, Passivity, Time-varying delay.

\section{Introduction}

Teleoperation system can extend human sensing, decision making and manipulation capabilities to a remote object and make the control system more flexible than a fully automated system. Such systems have been deployed in various domains ranging from space exploration, underwater operation, and nuclear reactor to minimally invasive surgery [21]. A traditional teleoperation system commonly consists of the human operator, the master robot, communication channels, the slave robot and the remote environment [22]. The operator drives the master robot to interact with the slave robot through communication channels. Since information produced during interaction between the slave and the force signals containing environmental information is fed back to the master side, system stability may be adversely affected. Therefore, achieving an optimal trade-off between stability and transparency is a challenge in bilateral teleoperation system. Teleoperation systems are also very sensitive to time delays, and the existence of even a small time delay may destabilize the whole system.

The idea of passivity characterized by mechanical energy, which uses force and velocity as efforts and flow variables, is an effective tool for establishing stability of bilateral teleoperation interaction [23]-[24]. Wave variable is a classic passivity-based method that can easily accommodate time delays. However, most of the wave-based systems lack the capability of to dealing with the time-varying delays issues. Several wave-based approaches are proposed to guarantee passivity under time varying delays [25]. Nonetheless, these methods are usually used to deal with the worst-case scenario by over-dissipating energy to the extent that transparency is largely decreased.

Hannaford et al. design an energy-based Time Domain Passivity Approach (TDPA) to adaptively dissipate energy [26]. [27] and [30] extend this method to deal with the time-varying delay issues. [31] and [19] add a virtual environment model to the energybased TDPA in order to achieve stable force perception under constant time delays. [18] combines the four-channel architecture with the energy-based TDPA to enhance transparency under constant time delays. The energy-based TDPA is simplified to a power-based TDPA in [28] which can dissipate the active energy generated in communication channels. The power-based TDPA proposed in [28] is extended in [29] to deal with the position drift issues. However, although the above TDPA-based system can guarantee system stability under time-varying delays, transparency degradation is their main drawback, even in the presence of small constant or no time delay. In [20], we design a new wave-based TDPA to significantly enhance the system transparency compared with the previous approaches in the presence of arbitrary time delays. However, our wave-based TDPA in [20] is only proposed to guarantee the passivity of communication channels. In practice, the control algorithms in the master side and the slave sides can also insert extra energy into the system especially when the measured external force signals are involved, to the extent that the overall system's passivity may still be easily jeopardized without proper control parameters. Further exploration for the TDPA based control researches is still needed.

Traditionally, the transient-state control performance is the main criterion in a control system [1]. Ilchmann et al. further analyse the tracking control for the nonlinear system and introduces the prescribed performance control (PPC) to obtain the prescribed desired transient behaviour [2]. This approach which is later studied by other research groups. The principal purpose of the PPC is to restrict the boundary of the tracking error to a randomly predefined small residual set with a convergence speed below a prescribed value, exhibiting a maximum overshoot less than a prescribed specified constant [3]. Xie et al. propose a new control algorithm combined with neural network for a robot system to assure the system's tracking error to be restricted by a prescribed decreasing boundary [4]. Bechlioulis et al. proposes a new neuroadaptive force/position control algorithm [5]. The performance of error evolution within prescribed bounds in both problems of regulation and tracking in robotic system is achieved in [6] Kostarigka et al. analyse the pre-set performance control problem for a flexible joint robot with unknown and possibly variable elasticity [7]. In the bilateral teleoperation research, Yang et al firstly apply the PPC to enhance the position synchronization performance [8]-[9] of the master and the slave robots. However, their approach primitively primarily has contains two major shortcomings. First, their proposed PPC approaches methods can only guarantee the position synchronization in the presence of constant time delays. Secondly, they only consider the scenario case of free space movement without the effect of human and environmental forces is considered. In [10], they treat the external forces as disturbances that are eliminated by using fuzzy logic controllers, whereas in the real application in the environmental contact, the external forces are non-removal. In fact, force tracking also plays a crucial role in the application of bilateral teleoperation which can largely enhance perception of the operator to of the remote environment. To our best knowledge, PPC has not been deployed into achieve force tracking forces in bilateral teleoperation systems.

The contributions of this paper are list as follows:

1) Unlike the previous system in [20] in which the TDPA is used to guarantee the passivity of communication channels, 
the new designed TDPA is able to guarantee the passivity of the whole system.

2) The previous TDPA system in [20] has a main drawback. That is, it has large tracking errors when the rate of time delays is largely increased. The new designed system can suppress the tracking errors no larger than the pre-set values by applying PPC.

3) Unlike the previous approaches, the extended PPC now can synchronize not only position, but also velocity and force. Moreover, the proposed PPC algorithm can be applied under arbitrary time-varying delays.

The remainder of the paper is list structured as follows: In Section 2 introduces so preliminary knowledge for an overview of the nonlinear dynamics of a system, and the characteristics of PPC and the wave-based TDPA is provided. The proposed system and the related control laws are introduced in Section 3. Section 4 describes the characteristics of the proposed control algorithms and its stability analysis. Section 5 presents the results of the some experimental results work conducted for with different time delays. Some conclusions are drawn in Section 6.

\section{Problem Formulation}

\subsection{Dynamics of a teleoperation system}

The dynamics of the bilateral teleoperation system in this paper is modelled as:

$M_{m}\left(q_{m}\right) \ddot{q}_{m}+C_{m}\left(q_{m}, \dot{q}_{m}\right) \dot{q}_{m}+g_{m}\left(q_{m}\right)+F_{m} \dot{q}_{m}+$

$f_{c m}\left(\dot{q}_{m}\right)=\tau_{m}+\tau_{h}$

$M_{s}\left(q_{s}\right) \ddot{q}_{s}+C_{s}\left(q_{s}, \dot{q}_{s}\right) \dot{q}_{s}+g_{s}\left(q_{s}\right)+F_{s} \dot{q}_{s}+f_{c s}\left(\dot{q}_{s}\right)=\tau_{s}-\tau_{e}$

where $i=m, s$ represent the master and slave. $\ddot{q}_{i}, \dot{q}_{i}, q_{i} \in R^{n}$ stand for the joint acceleration, velocity and position signals, respectively. $M_{i}\left(q_{i}\right) \in R^{n \times n}$ and $C_{i}\left(q_{i}, \dot{q}_{i}\right) \in R^{n \times n}$ are the inertia matrices and Coriolis/centrifugal effects, respectively. $g_{i}\left(q_{i}\right) \in R^{n}$ are the gravitational forces and $\tau_{i}$ are the control signals. $F_{i} \dot{q}_{i}(t)$ denote the viscous friction and $f_{c i}\left(\dot{q}_{i}(t)\right)$ denote the Coulomb friction. $\tau_{h}$ and $\tau_{e}$ are the real external human and environmental torques.

Some fundamental properties of the above nonlinear dynamic models in this paper are as follows [11]-[15]:

$\mathrm{P} 1$ : The inertia matrices $\mathrm{M}_{\mathrm{i}}\left(\mathrm{q}_{\mathrm{i}}\right)$ for a manipulator are symmetric positive-definite which verify: $0<\sigma_{\min }\left(M_{i}\left(q_{i}(t)\right)\right) I \leq$ $M_{i}\left(q_{i}(t)\right) \leq \sigma_{\max }\left(M_{i}\left(q_{i}(t)\right)\right) I \leq \infty$, and $I \in R^{n \times n}$ is the identity matrix. $\sigma_{\min }$ and $\sigma_{\max }$ are the strictly positive minimum and maximum eigenvalue of $M_{i}$ for $\mathrm{q}_{\mathrm{i}}$.

P2: Under an appropriate definition of the Coriolis/centrifugal matrix, the matrix $\dot{M}_{i}-2 C_{i}$ is skew symmetric and it can also be written as:

$$
\dot{M}_{i}\left(q_{i}(t)\right)=C_{i}\left(q_{i}(t), \dot{q}_{i}(t)\right)+C_{i}^{T}\left(q_{i}(t), \dot{q}_{i}(t)\right)
$$

P3: The Lagrangian dynamics are linearly parameterizable. That is:

$$
M_{i}\left(q_{i}\right) \ddot{q}_{i}+C_{i}\left(q_{i}, \dot{q}_{i}\right) \dot{q}_{i}+g_{i}\left(q_{i}\right)=Y_{i}\left(q_{i}, \dot{q}_{i}, \ddot{q}_{i}\right) \theta_{i}
$$

where $\theta_{i}$ is a constant p-dimensional vector of inertia parameters and $Y_{i}\left(q_{i}, \dot{q}_{i}, \ddot{q}_{i}\right) \in R^{n \times p}$ is the matrix of known functions of the generalized coordinates and their higher derivatives [20].
P4: For the robots with revolute joints, there exists a positive $L$ bounding the Coriolis/centrifugal matrix as:

$\left\|C_{i}\left(q_{i}(t), x(t)\right) y(t)\right\|_{2} \leq L\|x(t)\|_{2}\|y(t)\|_{2}$

We define the synchronization variables as (6)-(7) where $\delta, k_{m}$, $k_{s}$ are positive constants. $\tau_{h}^{*}$ and $\tau_{e}^{*}(t)$ are the measured human and environmental signals using force observers.

$$
\begin{aligned}
& r_{m}(t)=\dot{q}_{m}(t)+\delta q_{m}(t)-k_{m} \tau_{h}^{*}(t) \\
& r_{s}(t)=\dot{q}_{s}(t)+\delta q_{s}(t)+k_{s} \tau_{h}^{*}(t)
\end{aligned}
$$

With the variables in (6)-(7) and Property 3 , the system models in (1)-(2) can be rewritten as:

$$
\begin{aligned}
& M_{m}\left(q_{m}\right) \dot{r}_{m}+C_{m}\left(q_{m}, \dot{q}_{m}\right) r_{m}=\tau_{m}+\tau_{h}+f_{m} \\
& M_{s}\left(q_{s}\right) \dot{r}_{s}+C_{s}\left(q_{s}, \dot{q}_{s}\right) r_{s}=\tau_{s}-\tau_{e}+f_{s}
\end{aligned}
$$

$f_{i}$ in (8) and (9) denote

$f_{m}=M_{m}\left(q_{m}\right)\left(\delta \dot{q}_{m}-k_{m} \dot{\tau}_{h}^{*}\right)+C_{m}\left(q_{m}, \dot{q}_{m}\right)\left(\delta q_{m}-k_{m} \tau_{h}^{*}\right)-$

$g_{m}\left(q_{m}\right)-F_{m} \dot{q}_{m}-f_{c m}\left(\dot{q}_{m}\right)$

$f_{s}=M_{s}\left(q_{s}\right)\left(\delta \dot{q}_{s}+k_{s} \dot{\tau}_{e}^{*}\right)+C_{s}\left(q_{s}, \dot{q}_{s}\right)\left(\delta q_{s}+k_{s} \tau_{s}^{*}\right)-g_{s}\left(q_{i}\right)-$

$F_{s} \dot{q}_{s}-f_{c s}\left(\dot{q}_{s}\right)$

The primitive objective of a bilateral teleoperation is to have the positions of the slave accurately synchronized to the position of the master. In the presence of time-varying delays, the position and velocity synchronization errors between the master and the slave can be defined as follows:

$e_{p m}(t)=q_{s}\left(t-T_{2}(t)\right)-q_{m}(t), e_{v m}(t)=\dot{q}_{s}\left(t-T_{2}(t)\right)-$ $\dot{q}_{m}(t)$

$e_{p s}(t)=q_{m}\left(t-T_{1}(t)\right)-q_{s}(t), e_{v s}(t)=\dot{q}_{m}\left(t-T_{1}(t)\right)-$ $\dot{q}_{s}(t)$

$T_{1}(t)$ and $T_{2}(t)$ are the forward and backward time delays.

Remark 1. In this paper, the differentials of unsymmetrical time delays $T_{1}(t)$ and $T_{2}(t)$ are bounded by $\bar{\mu}_{1,2}$. That is, $\left|\dot{\mathrm{T}}_{1,2}(\mathrm{t})\right| \leq \bar{\mu}_{1,2} \cdot \bar{\mu}_{1,2}$ are arbitrary positive constants. The timevarying delays $T_{1,2}(t)$ have their upper bounds $T_{1,2}^{\max }$. That is, $T_{1,2}(t) \leq T_{1,2}^{\max }$.

A secondary objective of the bilateral teleoperation is that the operator can genuinely feel the remote environment, which is predicated on the accurate force tracking between the two robots. In this paper, the measured human and environmental torques are modeled as (14)-(15) which contains position, velocity, the measured force signals including acceleration signals as well as the unmeasurable noises plus model errors [16]-[17].

$$
\begin{aligned}
\tau_{h}(t) & =\xi_{m}(t)-\alpha_{m} r_{m}(t)-\alpha_{m}^{\prime} \dot{r}_{m}(t) \\
\tau_{e}(t) & =\xi_{s}(t)+\alpha_{s} r_{s}(t)+\alpha_{s}^{\prime} \dot{r}_{s}(t)
\end{aligned}
$$

where $\alpha_{\mathrm{i}}$ are unknown positive gains. $\xi_{i}(t)$ are variables containing active parts of external forces, unmeasurable noises and model errors. The measurable torque tracking errors are defined as (16)-(17).

$$
\begin{array}{r}
e_{t m}(t)=k_{m} \tau_{h}^{*}(t)+k_{s} \tau_{e}^{*}\left(t-T_{2}(t)\right) \\
e_{t s}(t)=-k_{m} \tau_{h}^{*}\left(t-T_{1}(t)\right)-k_{s} \tau_{e}^{*}(t)
\end{array}
$$


According to the position, velocity and torque tracking errors $e_{p i}$, $e_{v i}$ and $e_{t i}$ as well as the defined variables in (6) and (7), we can define the total errors for the tracking of the transmission signals:

$e_{m}(t)=e_{v m}(t)+\delta e_{p m}(t)+e_{t m}(t)=r_{s}\left(t-T_{2}(t)\right)-r_{m}(t)$

$e_{s}(t)=e_{v s}(t)+\delta e_{p s}(t)+e_{t s}(t)=r_{m}\left(t-T_{1}(t)\right)-r_{s}(t)$

\subsection{Fuzzy Logic Control}

Fuzzy logic control algorithms can be used to generally estimate the dynamic uncertainties. A fuzzy system is a collection of fuzzy IF-THEN rules of the form

$$
R^{k}: I F z_{1} \text { is } A_{1}^{k} \text { and ... and } z_{n} \text { is } A_{n}^{k} \text {,THEN } y \text { is } B^{k}
$$

where $z=\left[z_{1}, z_{2}, \ldots, z_{n}\right]^{T} \in \Omega_{z}$ and $y \in \Omega_{y}$ are the linguistic variables associated with the input and output of the fuzzy logic controllers, respectively. $R^{k}$ is the k-th rule. Fuzzy sets $A_{1}^{k} \ldots A_{n}^{k}$ and $B^{k}$ are associated with the membership functions $\mu_{A_{l}^{k}}\left(z_{l}\right)$ and $\mu_{B^{k}}$. The strategy of singleton fuzzification, product inference and center-average defuzzification are used while the output is:

$y(z(t))=\frac{\sum_{k=1}^{m} y^{k}\left(\prod_{l=1}^{n} \mu_{A_{l}^{k}}\left(z_{l}(t)\right)\right)}{\sum_{k=1}^{m} \prod_{l=1}^{n} \mu_{A_{l}^{k}}\left(z_{l}(t)\right)}$

where $y^{k}$ denotes the point in $R$ that $\mu_{B}$ can achieve its maximum value (assuming $\mu_{B}{ }^{k}\left(y^{k}\right)=1$ ). The fuzzy logic controller can be represented as (17) by introducing the concept of the fuzzy basic function vector $\varsigma(z(t))$ :

$y(z(t))=\theta^{T}(t) \varsigma(z(t))$

where the weight factor is $\theta(t)=\left[y^{1}(t), y^{2}(t), \ldots, y^{m}(t)\right]^{T}$ and the fuzzy basic function vector is $\varsigma(z(t))=$ $\left[\varsigma_{1}(z(t)), \varsigma_{2}(z(t)), \ldots, \varsigma_{m}(z(t))\right]$.

$\varsigma(z(t))=\frac{\prod_{l=1}^{n} \mu_{A_{l}^{k}}\left(z_{l}(t)\right)}{\sum_{k=1}^{m} \prod_{l=1}^{n} \mu_{A_{l}^{k}}\left(z_{l}(t)\right)}$

Based on the universal approximation theorem, the optimal approximation parameter $\theta^{*}$ exist and $\theta^{* T}(t) \varsigma(z(t))$ can estimate a nonlinear function $f(z(t))$ over a compact set to any degree of accuracy where $\theta^{*}$ can be defined as:

$\theta^{*}=\arg \min _{\theta \in \Omega_{\theta}}\left(\sup _{z \in \Omega_{z}}\left|\theta^{T}(t) \varsigma(z(t))-f(z(t))\right|\right)$

where $\Omega_{\theta}$ and $\Omega_{z}$ are the sets of suitable bounds on $\theta(t)$ and $z(t)$, respectively. The minimum approximation error satisfies

$f(z(t))=\theta^{* T} \varsigma(z(t))+\vartheta(z(t))$

and the positive constants $\epsilon^{*}$ exists such that $\|\epsilon(z(t))\| \leq \epsilon^{*}$ over the compact set $\Omega_{z}$. Due to the existing friction and backlash, the robots' dynamic functions can be seen as piecewise continuous functions. Supposing $G^{\prime}(z(t))$ is also a piecewise function where $f^{\prime}(z(t))=f_{1}(z(t))$ (continuous part) + $f_{2}(z(t))$ (bounded piecewise term). Therefore,

$f^{\prime}(z(t))=\theta^{* T} \varsigma(z(t))+\vartheta(z(t))+f_{2}(z(t))=\theta^{* T} \varsigma(z(t))+$ $\bar{\vartheta}(z(t))$

where $\bar{\epsilon}(z(t))=\vartheta(z(t))+f_{2}(z(t)) . \bar{\vartheta}^{*}$ is defined as an upper bound of the piecewise function approximation error $\bar{\vartheta}^{*} \geq$ $\|\bar{\vartheta}(z(t))\|$.

\subsection{Prescribed performance control}

The prescribed performance consists of the minimum convergence velocity, the maximum steady state error and the maximum allowed overshoot are set to be a priori [8] Considering the tracking errors $e_{i}$ in (18)-(19), the prescribed performance is achieved if $e_{i}$ are restricted in a predefined region bounded by a decaying function of time. The prescribed performance can be expressed as:

$\begin{cases}-H_{i} A_{i}(t)<e_{i}(t)<A_{i}(t), & \text { if } e_{i}(0) \geq 0 \\ -A_{i}(t)<e_{i}(t)<H_{i} A_{i}(t), & \text { if } e_{i}(0) \leq 0\end{cases}$

where $0<H_{i}<1$ and $A_{i}(t)$ is the performance functions associated with $e_{i}(t)$.

$A_{i}(t)=\left(A_{i 0}-A_{i \infty}\right) e^{-L_{i} t}+A_{i \infty}$

where $A_{i 0}, A_{i \infty}$ and $L_{i}$ are positive constants. $A_{i 0}$ are the initial value of $A_{i}(t)$ that $A_{i 0}=A_{i}(0) . A_{i 0}$ determine the predefined constraint of the initial values of $e_{i}(t)$ that should be large enough. $A_{i \infty}=\lim _{t \rightarrow \infty} A_{i}(t)$ are the final predefined constraint of $A_{i}(t)$ that usually set to be small in order to limit the maximum values of $\dot{e}_{i}(t)$ in the steady state. $L_{i}$ are the minimum convergence velocities.

Define

$$
\epsilon_{i}(t)=R_{i}\left(\frac{e_{i}(t)}{A_{i}(t)}\right)
$$

where $\epsilon_{i}(t)$ is the error vector via transformation. $R_{i}$ is a smooth, strictly increasing function shown as follows:

$R_{i}\left(\frac{e_{i}(t)}{A_{i}(t)}\right)= \begin{cases}\ln \frac{\frac{e_{i}(t)}{A_{i}(t)}+H_{i}}{H_{i}-H_{i} \frac{e_{i}(t)}{A_{i}(t)}}, & \text { if } e_{i}(0) \geq 0 \\ \ln \frac{H_{i} \frac{e_{i}(t)}{A_{i}(t)}+H_{i}}{H_{i}-\frac{e_{i}(t)}{A_{i}(t)}}, & \text { if } e_{i}(0)<0\end{cases}$

The derivative of $\epsilon_{i}(t)$ can be written as:

$$
\dot{\epsilon}_{i}(t)=\partial R_{i}\left(\dot{e}_{i}(t)-\frac{\dot{A}_{i}(t) e_{i}(t)}{A_{i}(t)}\right)
$$

where $\partial R_{i}=\frac{\partial R_{i}}{\partial e_{i} / A_{i}} \frac{1}{A_{i}}$. Since $R_{i}$ is a smooth strictly increasing function and satisfies: $R_{i}:\left(-H_{i}, 1\right) \rightarrow(-\infty,+\infty), e_{i}(0) \geq 0$ and $R_{i}:\left(-1, H_{i}\right) \rightarrow(-\infty,+\infty), e_{i}(0)<0$ based on (26), by guaranteeing the boundedness of $\epsilon_{i}(t),(26)$ can be achieved. When $\epsilon_{i}(t)$ converges to zero, $e_{i}$ will also converge to zero to the extent that the accurate position, velocity and torque tracking can be achieved. The following inequalities are true.

$$
\left\{\begin{array}{c}
-L_{i}<\frac{\dot{A}_{i}(t)}{A_{i}(t)}<0 \\
\left|\epsilon_{i}(t)\right|>\frac{4}{\left(H_{i}+1\right) A_{i}(t)}\left|e_{i}(t)\right| \\
\partial R_{i}>\frac{4}{\left(H_{i}+1\right) A_{i}(t)}>\frac{4}{\left(H_{i}+1\right) A_{i 0}}>0 \\
e_{i}^{T}(t) \partial R_{i} \epsilon_{i}(t) \geq\left(\frac{4}{\left(H_{i}+1\right) A_{i 0}}\right)^{2}\left\|e_{i}\right\|^{2}
\end{array}\right.
$$




\subsection{Passivity of the overall system}

The master-slave teleoperation system can be described by the pairs of power-conjugated variables, force, position and velocity at each terminal. (32) describes the power flow $P(t)$ into the bilateral teleoperation as shown in Fig.1.

$$
\begin{aligned}
& P(t)=V_{\text {ou }}(t) I_{\text {in }}(t)-V_{\text {in }}(t) I_{\text {ou }}(t)= \\
& \underbrace{\left(V_{\text {ou }}(t) I_{\text {in }}(t)-V_{m}(t) I_{m}(t)\right)}_{P_{m}-\text { master }}+\underbrace{\left(V_{m}(t) I_{m}(t)-V_{s}(t) I_{s}(t)\right)}_{P_{\text {com }} \text {-comm. cha. }}+ \\
& \underbrace{\left(V_{s}(t) I_{s}(t)-V_{\text {in }}(t) I_{\text {ou }}(t)\right)}_{P_{s}-\text { slave }}
\end{aligned}
$$

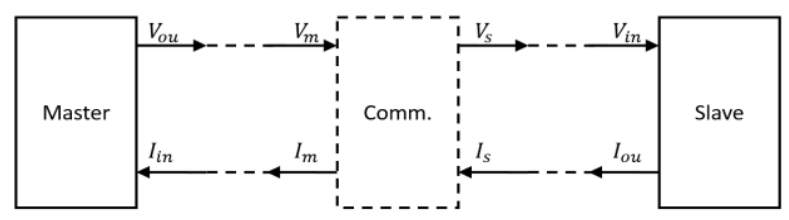

Fig.1. Power flow in a master-slave system

where $V_{o u}(t), I_{\text {in }}(t), V_{\text {in }}(t)$ and $I_{o u}(t)$ are output signal from the master, input signal into the master, input signal into the slave and output signal from the slave. $V_{m}(t), I_{m}(t), V_{s}(t)$ and $I_{s}(t)$ are the transmission control signals for the communication channels. From (30), only when the passivity of the master, the communication channels and the slave are simultaneously maintained, can the overall system's passivity be guaranteed.

In this paper, the passivity of communication channels can be guaranteed by using the wave-based TDPA proposed by the authors in [20]. The wave-based TDPA has the following advantages compared with previous approaches: 1) wave-based TDPA can accurately observe the power flow in the communication channels and provide higher transparency than traditional TDPA to the teleoperation system. 2) the wave transformation guarantees channels' passivity under constant time delays and provide accurate signal transmission without any biased terms. The applied wave transformation is introduced as Fig. 2 and the wave variables are given as (33)-(34)

$$
\begin{aligned}
& u_{m}(t)=\frac{b}{\sqrt{2 b}} V_{m}(t), u_{s}(t)=\frac{\left(b+\frac{b}{B}\right) V_{s}(t)+I_{s}(t)}{\sqrt{2 b}} \\
& v_{m}(t)=\frac{(B+1) I_{m}(t)-b V_{m}(t)}{\sqrt{2 b}}, v_{s}(t)=\frac{B}{\sqrt{2 b}} I_{s}(t)
\end{aligned}
$$

After transformation, the feed-forward and feedback signals transmission in the wave transformation can be derived as:

$$
\begin{aligned}
& V_{s}(t)=V_{m}\left(t-T_{1}(t)\right) \\
& I_{m}(t)=I_{s}\left(t-T_{2}(t)\right)
\end{aligned}
$$

Based on the given wave variables, the power flow into the communication channels can be expressed as:

$$
\begin{aligned}
& P_{c o m}(t)=V_{m}(t) I_{m}(t)-V_{s}(t) I_{s}(t)=\frac{2}{1+B} u_{m}^{T}(t) u_{m}(t)+ \\
& \frac{2}{M(1+B)} v_{m}^{T}(t) v_{m}(t)-\frac{1}{M(1+B)}\left(v_{m}(t)-M u_{m}(t)\right)^{T}\left(v_{m}(t)-\right. \\
& \left.M u_{m}(t)\right)-\frac{1}{M(1+B)} \dot{T}_{2} v_{m}^{T}(t) v_{m}(t)+\frac{2}{B(1+B)} v_{s}^{T}(t) v_{s}(t)+ \\
& \frac{2 M}{1+B} u_{s}^{T}(t) u_{s}(t)-\frac{1}{M(1+B)}\left(v_{s}(t)+M u_{s}(t)\right)^{T}\left(v_{s}(t)+\right. \\
& \left.M u_{s}(t)\right)-\frac{M}{1+B} \dot{T}_{1} u_{s}^{T}(t) u_{s}(t)+ \\
& \frac{d}{d t} \frac{M}{1+B} \int_{t-T_{1}(t)}^{t} u_{m}^{T}(\eta) u_{m}(\eta) d \eta+ \\
& \frac{d}{d t} \frac{1}{M(1+B)} \int_{t-T_{2}(t)}^{t} v_{s}^{T}(\eta) v_{s}(\eta) d \eta=P_{d i s s}(t)+\frac{d E}{d t}(t) \\
& E(t)=\frac{M}{1+B} \int_{t-T_{1}(t)}^{t} u_{m}^{T}(\eta) u_{m}(\eta) d \eta+ \\
& \frac{1}{M(1+B)} \int_{t-T_{2}(t)}^{t} v_{s}^{T}(\eta) v_{s}(\eta) d \eta \\
& P_{d i s s}(t)=\frac{2}{1+B} u_{m}^{T}(t) u_{m}(t)+\frac{2}{M(1+B)} v_{m}^{T}(t) v_{m}(t)- \\
& \frac{1}{M(1+B)}\left(v_{m}(t)-M u_{m}(t)\right)^{T}\left(v_{m}(t)-M u_{m}(t)\right)- \\
& \frac{1}{M(1+B)} \dot{T}_{2} v_{m}^{T}(t) v_{m}(t)+\frac{2}{B(1+B)} v_{s}^{T}(t) v_{s}(t)+\frac{2 M}{1+B} u_{s}^{T}(t) u_{s}(t)- \\
& \frac{1}{M(1+B)}\left(v_{s}(t)+M u_{s}(t)\right)^{T}\left(v_{s}(t)+M u_{s}(t)\right)- \\
& \frac{M}{1+B} \dot{T}_{1} u_{s}^{T}(t) u_{s}(t)
\end{aligned}
$$

where $\mathrm{M}$ is a positive constant which relates the unit of $u_{m, s}$ and $v_{m, s}$. According to (38), the net energy flow is absolutely positive to guarantee passivity of the communication channels. Based on the definition of passivity and assumption of $E(0)=0$, the energy flow from master to slave is derived as:

$$
\begin{aligned}
& E_{\text {flow }}(t)=\int_{0}^{t} P(\eta) d \eta=\int_{0}^{t}\left(P_{\text {diss }}(\eta)+\frac{d E}{d t}(\eta)\right) d \eta=E(t)- \\
& E(0)+\int_{0}^{t} P_{\text {diss }}(\eta) d \eta \geq \int_{0}^{t} P_{\text {diss }}(\eta) d \eta
\end{aligned}
$$

Therefore, under the condition that $P_{\text {diss }}(\eta) \geq 0$, based on (40), the energy flow $E_{\text {flow }}(t)$ is more than zero and the passivity of the communication channels can be achieved.

\section{Control algorithm design}

The following controller design is based on the above passivity analysis. From (35)-(38), we can deduct that by designing new controllers to guarantee (37) to be non-negative, the passivity of the overall system can be achieved. Based on (37), we can design the passivity observers for the communication channels as:

$$
\begin{aligned}
& P_{c m}(t)=\frac{2}{1+B} u_{m}^{T}(t) u_{m}(t)+\frac{2}{M(1+B)} v_{m}^{T}(t) v_{m}(t)- \\
& \frac{1}{M(1+B)}\left(v_{m}(t)-M u_{m}(t)\right)^{T}\left(v_{m}(t)-M u_{m}(t)\right)- \\
& \frac{1}{M(1+B)} \varepsilon_{2} v_{m}^{T}(t) v_{m}(t)
\end{aligned}
$$

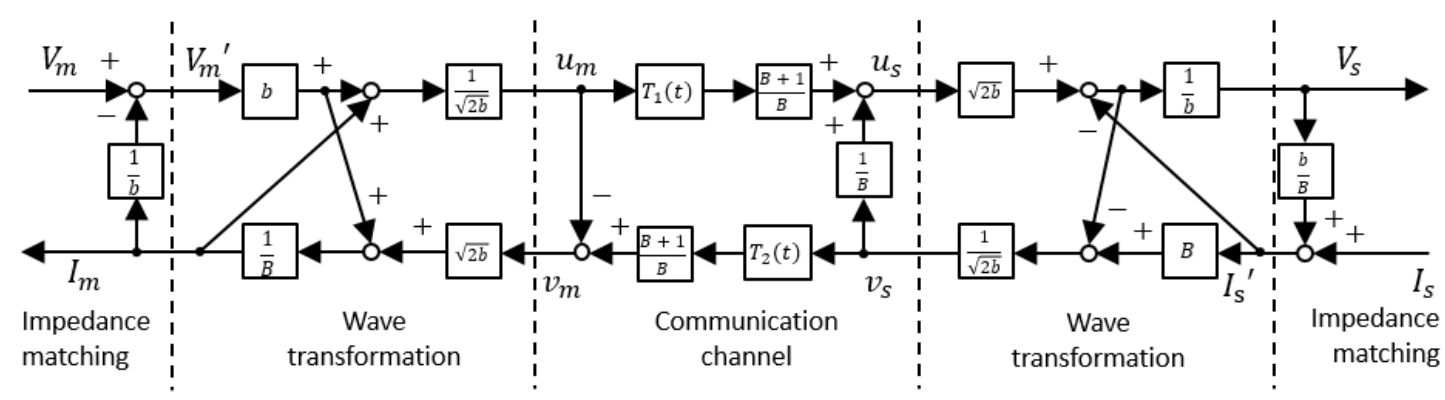

Fig.2. Proposed wave transformation 


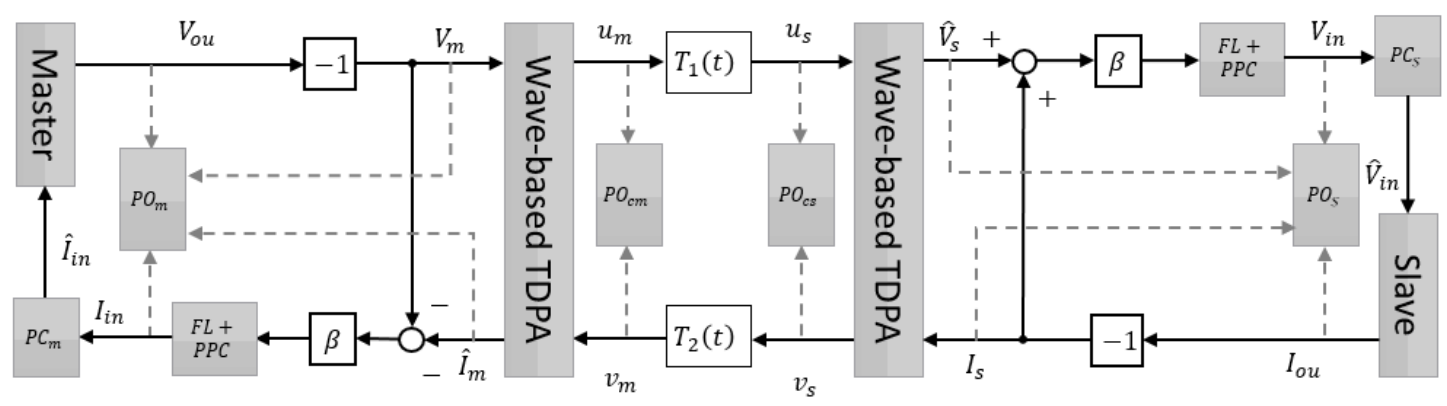

Fig.3. Total block diagram

$P_{c S}(t)=\frac{2}{B(1+B)} v_{s}^{T}(t) v_{s}(t)+\frac{2 M}{1+B} u_{s}^{T}(t) u_{s}(t)-\frac{1}{M(1+B)}\left(v_{s}(t)+\right.$ $\left.M u_{s}(t)\right)^{T}\left(v_{s}(t)+M u_{s}(t)\right)-\frac{M}{1+B} \varepsilon_{1} u_{s}^{T}(t) u_{s}(t)$

Based on (41) and (42), since $P_{c m}(t)$ and $P_{c s}(t)$ only have the information observed at the master and slave terminals, respectively, $P_{c m}(t)$ and $P_{c s}(t)$ can observe the power dissipation components in real time. The proposed wave transformation aims to guarantee the passivity of the communication channels in the presence of constant delays. When $\dot{T}_{1,2} \geq 0, P_{c m}(t)$ and $P_{c s}(t)$ are required to observe non-negative power information so that the passivity controllers will not be launched to affect the system transparency. By setting

$$
\left\{\begin{array}{l}
2 \sqrt{\frac{(2-M)}{M}} \geq 2 \Rightarrow 1 \geq M>0 \\
\frac{2}{B}-\frac{1}{M} \geq 0 \Rightarrow 2 M \geq B>1
\end{array}\right.
$$

$P_{c m j}(t)$ and $P_{c s j}(t)$ can surely observe non-negative power information when $\dot{T}_{1,2} \geq 0 . \varepsilon_{1,2}$ in (39)-(40) are the preset constants denoting the upper bound of the differential of time delays. The total block diagram of the proposed system is shown in Fig.3, where

$V_{\text {ou }}(t)=-r_{m}(t), I_{\text {ou }}(t)=r_{s}(t)$

$V_{m}(t)=-V_{\text {ou }}(t), I_{s}(t)=-I_{\text {ou }}$

$V_{m}(t)$ and $I_{s}(t)$ are transmitted via the wave transformation in Fig.2 for their further application in PPC controllers. Applying the passivity observers, we design the passivity controllers for the communication channels as:

$$
\begin{aligned}
& \hat{I}_{m}(t)=I_{m}(t)+\Gamma_{c m}(t) V_{m}(t) \\
& \hat{V}_{s}(t)=V_{s}(t)+\Gamma_{c s}(t) I_{s}(t)
\end{aligned}
$$

where

$$
\begin{aligned}
& \Gamma_{c m}(t)=\left\{\begin{array}{c}
0, \text { if } P_{c m}(t) \geq 0 \\
-P_{c m}(t)\left(V_{m}^{T}(t) V_{m}(t)\right)^{-1}, \text { else, if }\left|V_{m}(t)\right|>0
\end{array}\right. \\
& \Gamma_{c s}(t)=\left\{\begin{array}{c}
0, \text { if } P_{c s}(t) \geq 0 \\
P_{c s}(t)\left(I_{s}^{T}(t) I_{s}(t)\right)^{-1}, \text { else, if }\left|I_{s}(t)\right|>0
\end{array}\right.
\end{aligned}
$$

Moreover, to observe the power flow on the master and slave sides, we also design the passivity observers to be:

$$
\begin{aligned}
& P_{m}(t)=V_{\text {ou }}(t) I_{\text {in }}(t)-V_{m}(t) I_{m}(t) \\
& P_{s}(t)=V_{s}(t) I_{s}(t)-V_{\text {in }}(t) I_{\text {ou }}(t)
\end{aligned}
$$

The passivity controllers for the master and the slave side are designed as:

$$
\begin{aligned}
& \hat{I}_{\text {in }}(t)=I_{\text {in }}(t)+\Gamma_{m}(t) V_{\text {ou }}(t) \\
& \hat{V}_{\text {in }}(t)=V_{\text {in }}(t)+\Gamma_{s}(t) I_{\text {ou }}(t)
\end{aligned}
$$

where

$$
\Gamma_{m}(t)=\left\{\begin{array}{c}
0, \text { if } P_{m}(t) \geq 0 \\
\left(-P_{m}(t)+\Gamma_{c m}(t) V_{m}^{T}(t) V_{m}(t)\right)\left(V_{o u}^{T}(t) V_{\text {ou }}(t)\right)^{-1} \\
\text { else, if }\left|V_{\text {ou }}(t)\right|>0
\end{array}\right.
$$$$
\Gamma_{s}(t)=\left\{\begin{array}{c}
0, \text { if } P_{s}(t) \geq 0 \\
\left(P_{s}(t)+\Gamma_{c s}(t) I_{s}^{T}(t) I_{s}(t)\right)\left(I_{\text {ou }}^{T}(t) I_{\text {ou }}(t)\right)^{-1} \\
\text { else, if }\left|I_{\text {ou }}(t)\right|>0
\end{array}\right.
$$

$\hat{I}_{\text {in }}(t)$ and $\hat{V}_{\text {in }}(t)$ can be seen as the final inputs to the master and the slave robots. Based on (47)-(48) and (53)-(54), it is noticeable that $\Gamma_{c m}(t) \geq 0, \Gamma_{c s}(t) \leq 0, \Gamma_{m}(t) \geq 0, \Gamma_{s}(t) \leq 0$.

Theorem 3.1. The designed passivity controllers (43)-(52) can ensure the passivity of the whole master-slave system in the presence of arbitrary time delays.

\section{Proof.}

$$
\begin{aligned}
& P^{*}(t)=V_{\text {ou }}(t) \hat{I}_{\text {in }}(t)-\hat{V}_{\text {in }}(t) I_{\text {ou }}(t) \\
& =\left(V_{\text {ou }}(t) \hat{I}_{\text {in }}(t)-V_{m}(t) \hat{I}_{m}(t)\right)+\left(V_{m}(t) \hat{I}_{m}(t)-\hat{V}_{s}(t) I_{s}(t)+\right. \\
& \left.V_{m}(t) \hat{I}_{m}(t)-\hat{V}_{s}(t) I_{s}(t)\right)+\left(\hat{V}_{s}(t) I_{s}(t)+\hat{V}_{s}(t) I_{s}(t)-\right. \\
& \left.\widehat{V}_{\text {in }}(t) I_{\text {ou }}(t)\right) \\
& =\left(V_{\text {ou }}(t)\left(I_{\text {in }}(t)+\Gamma_{m}(t) V_{\text {ou }}(t)\right)-V_{m}(t)\left(I_{m}(t)+\right.\right. \\
& \left.\left.\Gamma_{c m}(t) V_{m}(t)\right)\right)+\left(V_{m}(t)\left(I_{m}(t)+\Gamma_{c m}(t) V_{m}(t)\right)-\left(V_{s}(t)+\right.\right. \\
& \left.\left.\Gamma_{c s}(t) I_{s}(t)\right) I_{s}(t)\right)+\left(\left(V_{s}(t)+\Gamma_{c s}(t) I_{s}(t)\right) I_{s}(t)-\left(V_{\text {in }}(t)+\right.\right. \\
& \left.\left.\Gamma_{s}(t) I_{\text {ou }}(t)\right) I_{\text {ou }}(t)\right) \\
& =\left(P_{m}-\Gamma_{c m}(t) V_{m}^{T}(t) V_{m}(t)+\Gamma_{m}(t) V_{o u}^{T}(t) V_{\text {ou }}(t)\right)+\left(P_{c m}(t)+\right. \\
& \left.P_{c s}(t)+\frac{d E_{j}(t)}{d t}+\Gamma_{c m}(t) V_{m}^{T}(t) V_{m}(t)-\Gamma_{c s}(t) I_{s}^{T}(t) I_{s}(t)\right)+ \\
& \left(P_{s}+\Gamma_{c s}(t) I_{s}^{T}(t) I_{s}(t)-\Gamma_{s}(t) I_{o u}^{T}(t) I_{o u}(t)\right) \\
& =P_{m}^{*}(t)+P_{d i s s}^{*}(t)+\frac{d E}{d t}(t)+P_{s}^{*}(t)
\end{aligned}
$$

Based on the definitions of the passivity controllers, $P_{m}^{*}(t)$, $P_{\text {diss }}^{*}(t)$ and $P_{s}^{*}(t)$ are ensured to be non-negative, which is the 
sufficient condition for guaranteeing the passivity of the masterslave system.

Remark 2. From the above analysis, we can see that the designed passivity controllers are mainly used to reduce the values of position, velocity and torque signals to maintain system stability. Under large time varying delays, based on the values of the passivity observers, the position, velocity and torque control signals will be largely degraded, and large tracking errors occur. This is the main drawback of all of the TDPA-based systems. In this paper, we apply the PPC algorithm to restrict the tracking errors no larger than a preset value. The proposed TDPA in turn allows the PPC algorithm to be applied under time-varying delays, which also compensates for the drawback of [9] and [10]. In this way, the performance of the proposed system can be enlarged under sharply-varying delays while the stability is still guaranteed.

The outputs of the PPC controllers are given as:

$I_{p m}(t)=d_{m} \partial R_{m} \epsilon_{m}(t)$

$I_{p s}(t)=d_{s} \partial R_{s} \epsilon_{s}(t)$

$d_{m}$ and $d_{s}$ are positive definite diagonal matrices.

According to FLs approximation property, the functions $\hat{f}_{i}\left(Z_{i}\right)$ are applied in this paper to approximate $f_{i}$ in (10)-(11), $\xi_{i}$ in (14)-(15) with

$$
\hat{f}_{i}\left(Z_{i}\right)=\hat{\theta}_{i}^{T} \varsigma_{i}\left(Z_{i}\right)
$$

where $Z_{i}(t)=\left[\dot{q}_{i}^{T}(t), q_{i}^{T}(t)\right]^{T}, \hat{\theta}_{i}$ are the fuzzy adaptation parameters and $\varsigma_{i}\left(Z_{i}\right)$ are the vectors denoting the fuzzy basis functions. Define

$\tilde{f}_{i}\left(Z_{i}\right)=f_{i}+\xi_{i}+\chi_{i}-\hat{f}_{i}\left(Z_{i}\right)$

$\leq\left(\theta_{i}^{* T}-\hat{\theta}_{i}^{T}\right) \varsigma_{i}\left(Z_{i}\right)+\bar{\vartheta}_{i} r_{i}=\tilde{\theta}_{i}^{T} \varsigma_{i}\left(Z_{i}\right)+\bar{\vartheta}_{i} r_{i}$

Due to the piecewise continuous function $f_{c i}\left(\dot{q}_{i}\right), \bar{\vartheta}_{i}$ are the upper bound of $\vartheta_{i}\left(Z_{i}\right)$ and $f_{c i}\left(\dot{q}_{i}\right)$ associated with $r_{i}$.

By using the extended TDPA + PPC algorithms, the control laws can be finally designed as:

$\tau_{m}(t)=\beta\left(r_{s}\left(t-T_{2}(t)\right)-r_{m}(t)\right)-\beta \Gamma_{c m}(t) r_{m}(t)+$

$d_{m} \partial R_{m} \epsilon_{m}(t)-\Gamma_{m}(t) r_{m}(t)-\hat{f}_{m}\left(Z_{m}\right)$

$\tau_{s}(t)=\beta\left(r_{m}\left(t-T_{1}(t)\right)-r_{s}(t)\right)+\beta \Gamma_{c s 1}(t) r_{s}(t)+$

$d_{s} \partial R_{s} \epsilon_{s}(t)+\Gamma_{s}(t) r_{s}(t)-\hat{f}_{s}\left(Z_{s}\right)$

Compared with the TDPA in [20], [28]-[29] which can only guarantee the passivity of the communication channels, the proposed passivity observers and controllers in Fig. 3 can robustly guarantee the overall system's passivity (passivity of the master, the slave and the communication channels). Moreover, PPC is applied to enhance the signal synchronization. Unlike the PPC method in [8]-[9] which can only guarantee the position synchronization under constant time delays, the PPC applied in the Fig. 3 can simultaneously guarantee the synchronization of position, velocity and torque in the presence of time varying delays.

\section{Stability analysis}

This section analyzes the system's stability and position and torque tracking performances using Lyapunov functions.
Theorem 4.1. Considering the bilateral teleoperation described in (1)-(2), then with the human and environmental torques modeled as (14)-(15), the fuzzy logic laws are proposed as:

$$
\begin{aligned}
& \dot{\hat{\theta}}_{m}=\Lambda_{m} \zeta_{m}\left(Z_{m}\right)\left(r_{m}-r_{s}\left(t-T_{2}(t)\right)\right) \\
& \dot{\hat{\theta}}_{s}=\Lambda_{s} \varsigma_{s}\left(Z_{s}\right)\left(r_{s}-r_{m}\left(t-T_{1}(t)\right)\right)
\end{aligned}
$$

the errors of position, velocity and torque are bounded and the prescribed synchronization performance is guaranteed.

Proof. Consider a positive definite function $V=V_{1}+V_{2}+V_{3}$ for the system as:

$$
\begin{aligned}
& V_{1}=\frac{1}{2}\left[r_{m}^{T}(t) M_{m}\left(q_{m}\right) r_{m}(t)+r_{s}^{T}(t) M_{s}\left(q_{s}\right) r_{s}(t)+\right. \\
& \left.\operatorname{tr}\left(\tilde{\theta}_{m}^{T} \Lambda_{m}^{-1} \tilde{\theta}_{m}\right)+\operatorname{tr}\left(\tilde{\theta}_{s}^{T} \Lambda_{s}^{-1} \tilde{\theta}_{s}\right)\right] \\
& V_{2}=\frac{\beta}{2} \int_{t-T_{1}(t)}^{t} r_{m}^{T}(\eta) r_{m}(\eta) d \eta+\frac{\beta}{2} \int_{t-T_{2}(t)}^{t} r_{s}^{T}(\eta) r_{s}(\eta) d \eta+ \\
& \frac{\alpha_{m}^{\prime}}{2} r_{m}^{T}(t) r_{m}(t)+\frac{\alpha_{s}^{\prime}}{2} r_{s}^{T}(t) r_{s}(t) \\
& V_{3}=\frac{1}{2} \epsilon_{m}^{T} \epsilon_{m}+\frac{1}{2} \epsilon_{s}^{T} \epsilon_{s}
\end{aligned}
$$

The derivative of $V_{1}$ is given by

$$
\begin{aligned}
& \dot{V}_{1} \leq r_{m}^{T}(t)\left[\beta\left(r_{s}\left(t-T_{2}(t)\right)-r_{m}(t)\right)-\beta \Gamma_{c m}(t) r_{m}(t)-\right. \\
& \left.\Gamma_{m}(t) r_{m}(t)+\bar{\vartheta}_{m} r_{m}(t)-\alpha_{m} r_{m}(t)+\chi_{m} r_{m}(t)\right]+ \\
& r_{s}^{T}(t)\left[\beta\left(r_{m}\left(t-T_{1}(t)\right)-r_{s}(t)\right)+\beta \Gamma_{c s 1}(t) r_{s}(t)+\right. \\
& \left.\Gamma_{s}(t) r_{s}(t)+\bar{\vartheta}_{s} r_{s}(t)-\alpha_{s} r_{s}(t)+\chi_{s} r_{s}(t)\right]-\left(d_{m} e_{m}(t)+\right. \\
& \left.\dot{e}_{m}(t)\right) \partial R_{m} \epsilon_{m}(t)-\left(d_{s} e_{s}(t)+\dot{e}_{s}(t)\right) \partial R_{s} \epsilon_{s}(t)
\end{aligned}
$$

where $\chi_{i}$ are the positive constant that expressed as:

$$
\begin{gathered}
\chi_{m}=\sup \left\{\begin{array}{r}
\frac{1}{\left\|r_{m}(t)\right\|^{2}}\left(\left(d_{m} r_{m}(t)+d_{m} e_{m}(t)\right.\right. \\
\left.+\dot{e}_{m}(t)\right) \partial R_{m} \epsilon_{m}(t) \\
\left.\left.-\tilde{\theta}_{m}^{T} \varsigma_{m}\left(Z_{m}\right) r_{s}\left(t-T_{2}(t)\right)\right)\right\}
\end{array}\right. \\
\chi_{s}=\sup \left\{\frac { 1 } { \| r _ { s } ( t ) \| ^ { 2 } } \left(\left(d_{s} r_{s}(t)+d_{s} e_{s}(t)+\dot{e}_{s}(t)\right) \partial R_{s} \epsilon_{s}(t)\right.\right. \\
\left.\left.-\tilde{\theta}_{s}^{T} S_{s}\left(Z_{s}\right) r_{m}\left(t-T_{1}(t)\right)\right)\right\}
\end{gathered}
$$

The time derivatives of $V_{2}$ are given as

$$
\begin{aligned}
& \dot{V}_{2}=\frac{\beta}{2}\left(r_{m}^{T}(t) r_{m}(t)-r_{m}^{T}\left(t-T_{1}(t)\right) r_{m}\left(t-T_{1}(t)\right)+\right. \\
& \left.\dot{T}_{1}(t) r_{m}^{T}\left(t-T_{1}(t)\right) r_{m}\left(t-T_{1}(t)\right)\right)+\frac{\beta}{2}\left(r_{s}^{T}(t) r_{s}(t)-r_{s}^{T}(t-\right. \\
& \left.\left.T_{2}(t)\right) r_{s}\left(t-T_{2}(t)\right)+\dot{T}_{2}(t) r_{s}^{T}\left(t-T_{2}(t)\right) r_{s}\left(t-T_{2}(t)\right)\right)
\end{aligned}
$$

Applying (31), the derivative of $V_{3}$ can be expressed as

$$
\begin{aligned}
& \dot{V}_{3}=\epsilon_{m}^{T}(t) \dot{\epsilon}_{m}(t)+\epsilon_{s}^{T}(t) \dot{\epsilon}_{s}(t) \\
& =\epsilon_{m}^{T}(t) \partial R_{m}\left(\dot{e}_{m}(t)-\frac{\dot{A}_{m}(t) e_{m}(t)}{A_{m}(t)}\right)+\epsilon_{s}^{T}(t) \partial R_{s}\left(\dot{e}_{s}(t)-\right. \\
& \left.\frac{\dot{A}_{s}(t) e_{s}(t)}{A_{s}(t)}\right) \\
& \leq \epsilon_{m}^{T}(t) \partial R_{m} \dot{e}_{m}(t)+\epsilon_{m}^{T}(t) \partial R_{m} L_{m} e_{m}(t)+\epsilon_{s}^{T}(t) \partial R_{s} \dot{e}_{s}(t)+ \\
& \epsilon_{s}^{T}(t) \partial R_{s} L_{s} e_{s}(t)
\end{aligned}
$$

Constants $\sigma_{1,2}$ exist to make sure 


$$
\begin{aligned}
& \sigma_{1} \dot{T}_{1}(t) r_{m}^{T}(t) r_{m}(t) \geq \dot{T}_{1}(t) r_{m}^{T}\left(t-T_{1}(t)\right) r_{m}\left(t-T_{1}(t)\right) \\
& \sigma_{2} \dot{T}_{2}(t) r_{s}^{T}(t) r_{s}(t) \geq \dot{T}_{2}(t) r_{s}^{T}\left(t-T_{2}(t)\right) r_{s}\left(t-T_{2}(t)\right)
\end{aligned}
$$

From (63)-(70), $\dot{V}$ can be derived as:

$$
\begin{aligned}
& \dot{V} \leq-\left(\alpha_{m}+\beta \Gamma_{c m}(t)+\Gamma_{m}(t)-\frac{\beta \sigma_{1} \dot{T}_{1}(t)}{2}-\chi_{m}-\right. \\
& \left.\bar{\vartheta}_{m}\right) r_{m}^{T}(t) r_{m}(t)-\left(\alpha_{s}-\beta \Gamma_{c s}(t)-\Gamma_{s}(t)-\frac{\sigma_{2} \dot{T}_{2}(t) \beta}{2}-\chi_{s}-\right. \\
& \left.\bar{\vartheta}_{s}\right) r_{s}^{T}(t) r_{s}(t)-\frac{\beta}{2}\left(e_{v m}(t)+\delta e_{p m}(t)+e_{t m}(t)\right)^{T}\left(e_{v m}(t)+\right. \\
& \left.\delta e_{p m}(t)+e_{t m}(t)\right)-\frac{\beta}{2}\left(e_{v s}(t)+\delta e_{p s}(t)+e_{t s}(t)\right)^{T}\left(e_{v s}(t)+\right. \\
& \left.\delta e_{p s}(t)+e_{t s}(t)\right)-\left(d_{m}-L_{m}\right)\left(\frac{4}{\left(H_{m}+1\right) A_{m 0}}\right)^{2}\left\|e_{m}(t)\right\|^{2}- \\
& \left(d_{s}-L_{s}\right)\left(\frac{4}{\left(H_{s}+1\right) A_{s 0}}\right)^{2}\left\|e_{s}(t)\right\|^{2}
\end{aligned}
$$

By guaranteeing the first two terms of (71) to be non-positive, (71) then can be negative. That is,

$$
\begin{gathered}
\alpha_{m}+\beta \Gamma_{c m}(t)+\Gamma_{m}(t) \geq \frac{\beta \sigma_{1} \dot{T}_{1}(t)}{2}+\chi_{m}+\bar{\vartheta}_{m} \\
\alpha_{s}-\beta \Gamma_{c s}(t)-\Gamma_{s}(t) \geq \frac{\sigma_{2} \dot{T}_{2}(t) \beta}{2}+\chi_{s}+\bar{\vartheta}_{s}
\end{gathered}
$$

From (47)-(48) and (53)-(54), $\quad \Gamma_{c m}(t) \geq 0, \quad \Gamma_{c s}(t) \leq 0$, $\Gamma_{m}(t) \geq 0, \Gamma_{s}(t) \leq 0$. Theorem 3.1 proves that the Passivity controllers $\Gamma_{c m}(t) \Gamma_{m}(t), \Gamma_{c s}(t), \Gamma_{m}(t)$ can eliminate the energy produced by time delays and external disturbances, $\left(\frac{\beta \sigma_{1} \dot{T}_{1}(t)}{2}+\chi_{m}+\bar{\vartheta}_{m}\right.$ and $\left.-\frac{\sigma_{2} \dot{T}_{2}(t) \beta}{2}-\chi_{s}-\bar{\vartheta}_{s}\right)$, and guarantee the passivity of the overall system. Therefore, by setting a large value of $\beta$, (72) and (73) can be easily guaranteed to be true. To guarantee $\quad-\left(d_{m}-L_{m}\right)\left(\frac{4}{\left(H_{m}+1\right) A_{m 0}}\right)^{2}\left\|e_{m}(t)\right\|^{2}-\left(d_{s}-\right.$ $\left.L_{S}\right)\left(\frac{4}{\left(H_{S}+1\right) A_{s 0}}\right)^{2}\left\|e_{S}(t)\right\|^{2}$ to be negative definite, (74)-(75) should be satisfied.

$$
\begin{aligned}
& d_{m} \geq L_{m} \\
& d_{s} \geq L_{s}
\end{aligned}
$$

Therefore, $\dot{V}$ is guaranteed to be negative definite. Integrating both sides of (71), we get

$$
\begin{aligned}
& +\infty>V(0) \geq V(0)-V(t) \geq \int_{0}^{t}\left(\alpha_{m}+\beta \Gamma_{c m}(t)+\Gamma_{m}(t)-\right. \\
& \left.\frac{\beta \sigma_{1} \dot{T}_{1}(t)}{2}-\chi_{m}-\bar{\vartheta}_{m}\right) r_{m}^{T}(t) r_{m}(t)+\left(\alpha_{s}-\beta \Gamma_{c s}(t)-\Gamma_{s}(t)-\right. \\
& \left.\frac{\sigma_{2} \dot{T}_{2}(t) \beta}{2}-\chi_{s}-\bar{\vartheta}_{s}\right) r_{s}^{T}(t) r_{s}(t)+\frac{\beta}{2}\left(e_{v m}(t)+\delta e_{p m}(t)+\right. \\
& \left.e_{t m}(t)\right)^{T}\left(e_{v m}(t)+\delta e_{p m}(t)+e_{t m}(t)\right)+\frac{\beta}{2}\left(e_{v s}(t)+\right. \\
& \left.\delta e_{p s}(t)+e_{t s}(t)\right)^{T}\left(e_{v s}(t)+\delta e_{p s}(t)+e_{t s}(t)\right)+\left(d_{m}-\right. \\
& \left.L_{m}\right)\left(\frac{4}{\left(H_{m}+1\right) A_{m 0}}\right)^{2}\left\|e_{m}(t)\right\|^{2}+\left(d_{s}-L_{s}\right)\left(\frac{4}{\left(H_{s}+1\right) A_{s 0}}\right)^{2}\left\|e_{s}(t)\right\|^{2}
\end{aligned}
$$

Due to the positive definite storage functional $V$ and its negative definite differential $\dot{V}, \lim _{t \rightarrow \infty} V$ exists and is finite. Also, $\tilde{\theta}_{i}(t), \epsilon_{i}(t) \in L_{\infty}, \quad r_{i}, \quad, \quad e_{i} \in L_{\infty} \cap L_{2}$. The prescribed synchronization performance is obtained. Moreover, the position, velocity and torque synchronization errors asymptotically converge to zero when time tends to infinity based on the definition of PPC.

\section{Experimental results}

This section demonstrate a series of experiments to validate the proposed system. The applied experimental platform consists of two 3-DOF Phantom haptic devices: Phantom Omni and Phantom Desktop (Sensable Technologies, Inc., Wilmington, MA) as shown in Fig.4. During the experimental process, the control loop is configured as a $1 \mathrm{kHz}$ sampling rate. The Internet is applied as the communication channels in the experiment, and to tune the values of the time delays, extra Simulink time-delay models are also used. The wave impedance $\mathrm{b}$ is set as 2.5 . The impedances $\mathrm{B}$ and $\mathrm{M}$ are set as 2 and 1 , respectively. $\delta$ is set to be 1 . $k_{m}=k_{s}=1$. $\beta$ is set to be 3 . In the PPC controllers, $d_{m}=d_{s}=4 \quad . \quad L_{m}=L_{s}=0.2 \quad, \quad A_{m}(0)=A_{s}(0)=2$, $A_{m}(\infty)=A_{s}(\infty)=0.2$. The values of $A_{m}(\infty)$ and $A_{s}(\infty)$ determine all of the tracking errors will not exist 0.2. $\Lambda_{i}$ for the fuzzy control are set as 0.2 .
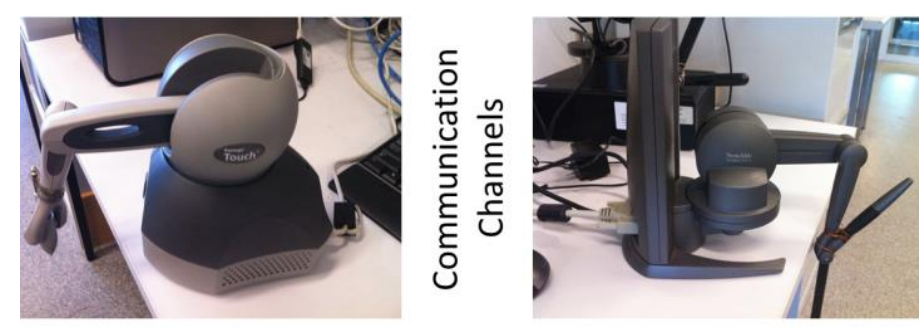

Fig.4. Experimental platform

\subsection{Slowly-varying delays}

In this section, the proposed system is compared with the conventional PD+d system in [17]. The time delays in this experiment are approximately $600 \mathrm{~s}$ with $100 \mathrm{~ms}$ variations, and the rate of the time delay is around 0.1 . In order to guarantee stability, the parameters in the system in [17] are required to satisfy $4 B_{r} B_{l}>\left(T_{1}^{\max ^{2}}+T_{2}^{\max ^{2}}\right) K_{l} K_{r}, B_{r} \geq B_{l}$ and $K_{r} \geq K_{l}$. Therefore, we set the differential gain $K_{d}=3$, and the proportional gains $K_{r}=K_{l}=3$. Hence, $B_{r}=B_{l}=1.5$. The PD+d system in [17] also uses the scaling gain $\sqrt{1-\bar{\mu}_{1,2}}$ for velocity transmission. It is noticeable that when $\bar{\mu}_{1,2} \geq 1$, this approach is too conservative and velocities cannot be transmitted. The first experiment demonstrates the two systems' performance during free motion. Figs. 5 shows the position and torque tracking and the related tracking errors of the system in [17] during free motion. Due to the large value of the velocity dampers, the operators can still feel large feedback force even without contacting any environmental object. The position tracking is also degraded by the velocity dampers. Also, the dynamic uncertainties, especially gravity model errors, influence the accuracy of position tracking of joint 2 and 3. Therefore, large position and torque tracking errors (around 0.5) are shown in Fig.5.

Figs. 6 and 7 show the position tracking, observed power signals and torque tracking of the proposed system. Under slowlyvarying delays, the power signals observed by the proposed observers are almost positive. Therefore, the proposed passivity controllers are not activated by the observers to degrade the system transparency. Moreover, the applied PPC method restrict the position errors to small values (less than 0.1 ) and the torque errors are also close to zero as show in Figs. 6 and 7. Therefore, the operator can hardly feel the force feedback during free motion. Although without dynamic models, the FL controllers are estimate the system uncertainties including gravity models so that 
the free motion is not affected by the gravity and highly accurate positon tracking can be achieved.

Then under the same time delays, the slave robots of the two systems are controlled to contact to a solid wall. Fig. 8 shows the position and torque signals and the related errors of the system in [17]. Since the operator can only feel the feedback signals from the PD controllers, and under large time delays, the velocity dampers severely affect the system transparency, tracking errors of the human and environmental torques exist during hard contact and large position errors appears in the steady state as shown in Fig.8.
Figs. 9 and 10 show the position, observer power signals and torque of the proposed system under hard contact. During hard contact, the observed torques are still almost positive, so that the proposed passivity controllers are nearly inactivated and the system's transparency is not affected by the passivity controllers. Since the environmental torques are directly feed back to the master side, highly accurate torque tracking is achieved. During the hard contact $(3 \mathrm{~s}-11 \mathrm{~s}$ in Fig.10), the torque tracking errors is zero. The output control signals of FL controllers are shown in Figure 11.
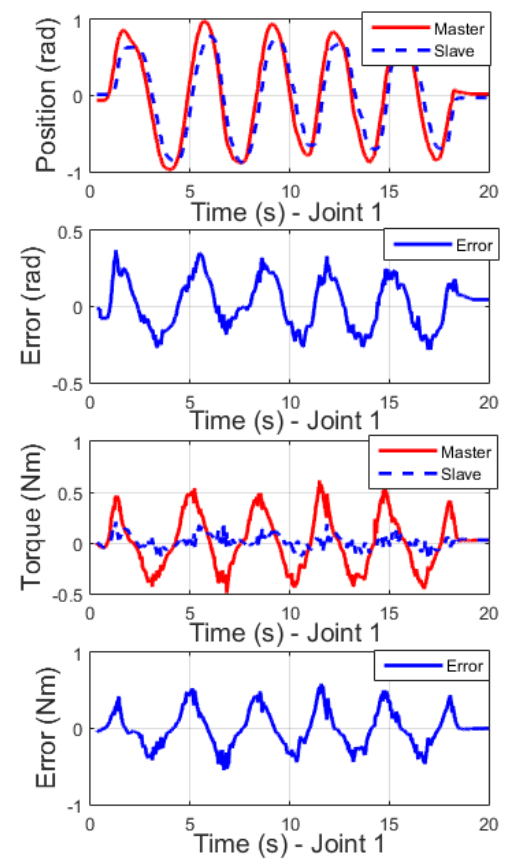
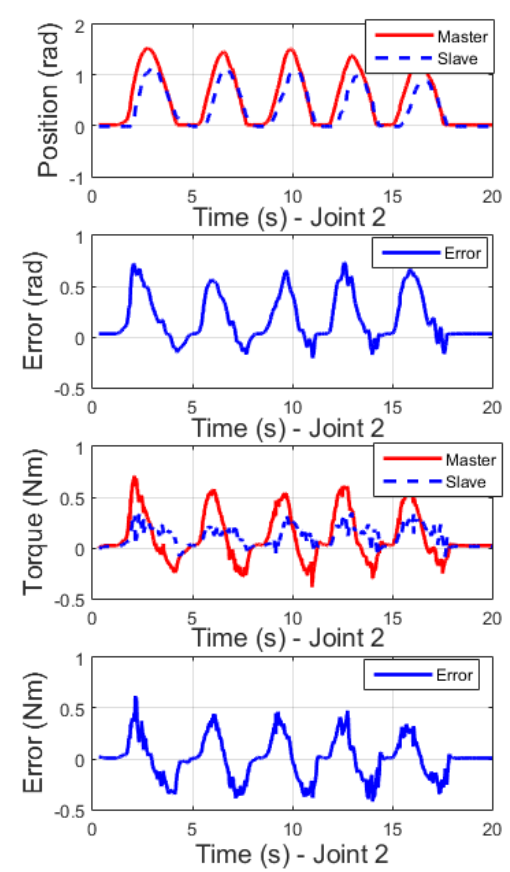
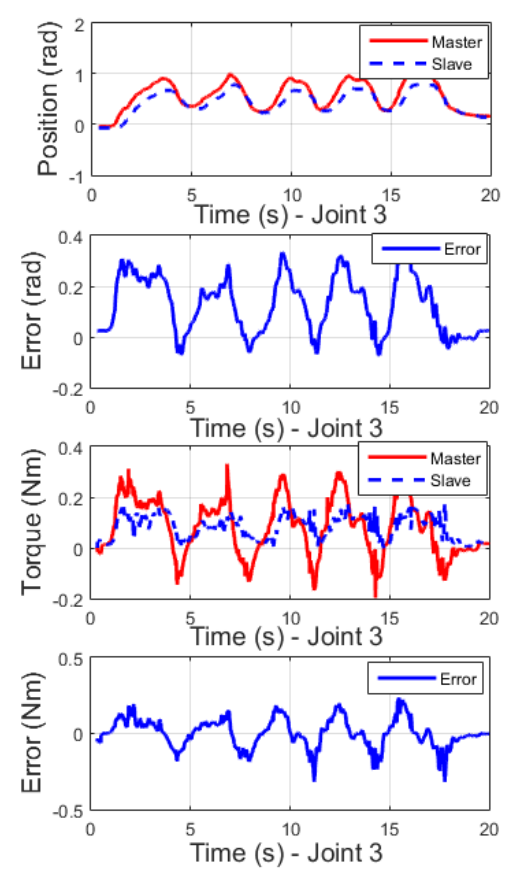

Fig.5. Position and torque tracking of the system in [17] during free motion (slowly-varying delays)
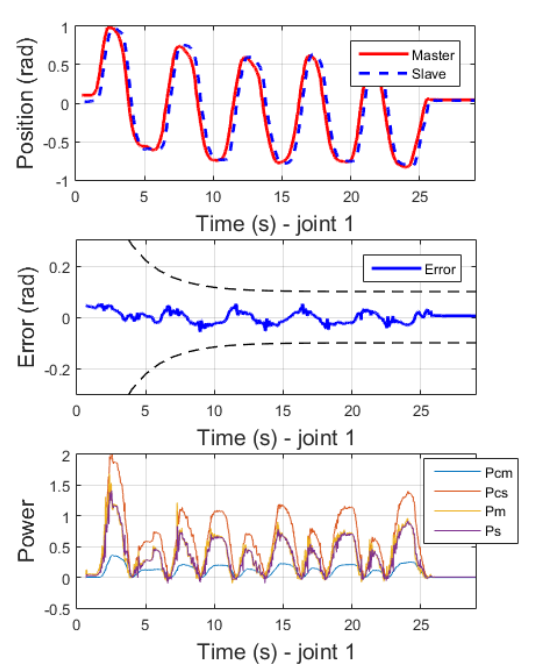
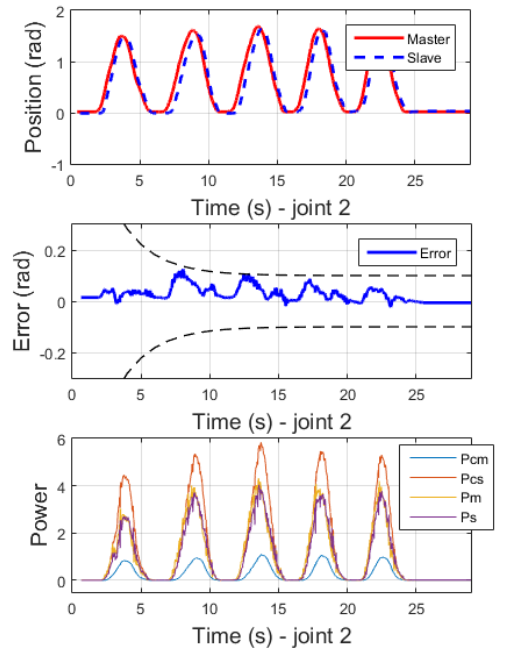
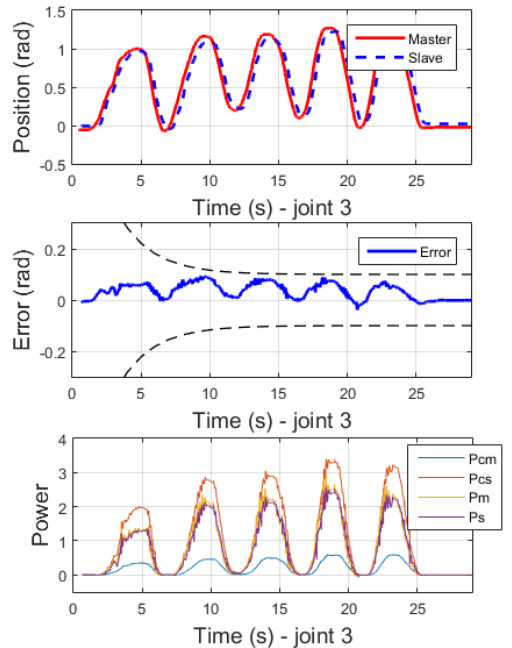

Fig.6. Position and observed power of our system during free motion (slowly-varying delays) 

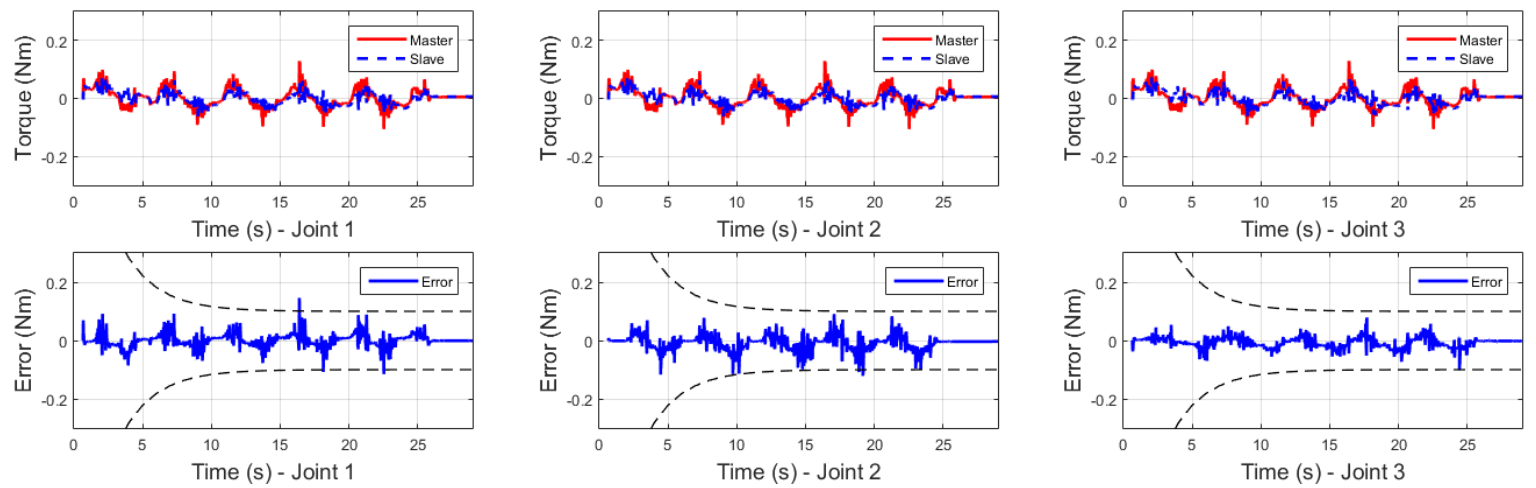

Fig.7. Torque of our system during free motion (slowly-varying delays)
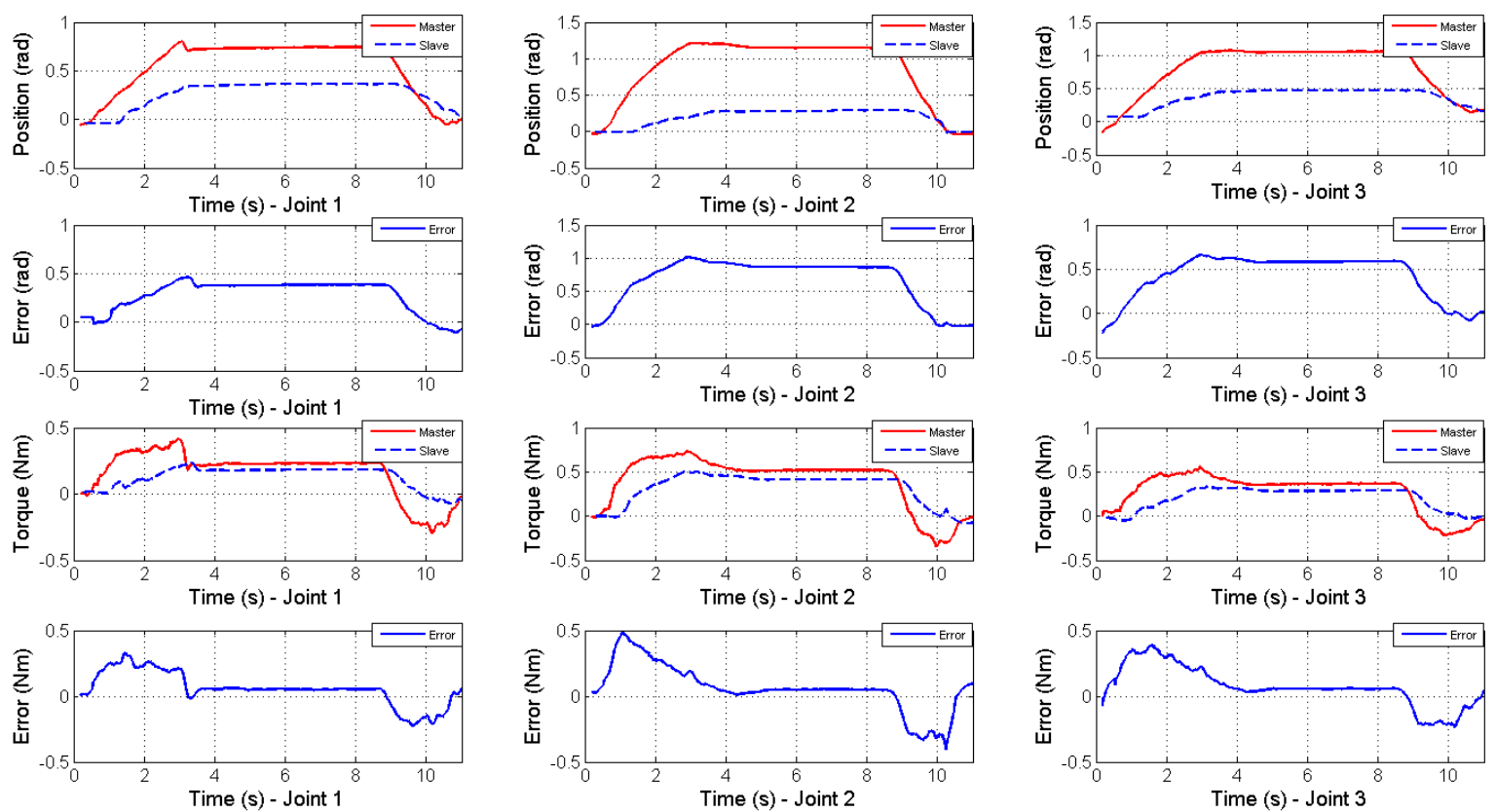

Fig.8. Position and torque tracking of the system in [17] during hard contact (slowly-varying delays)
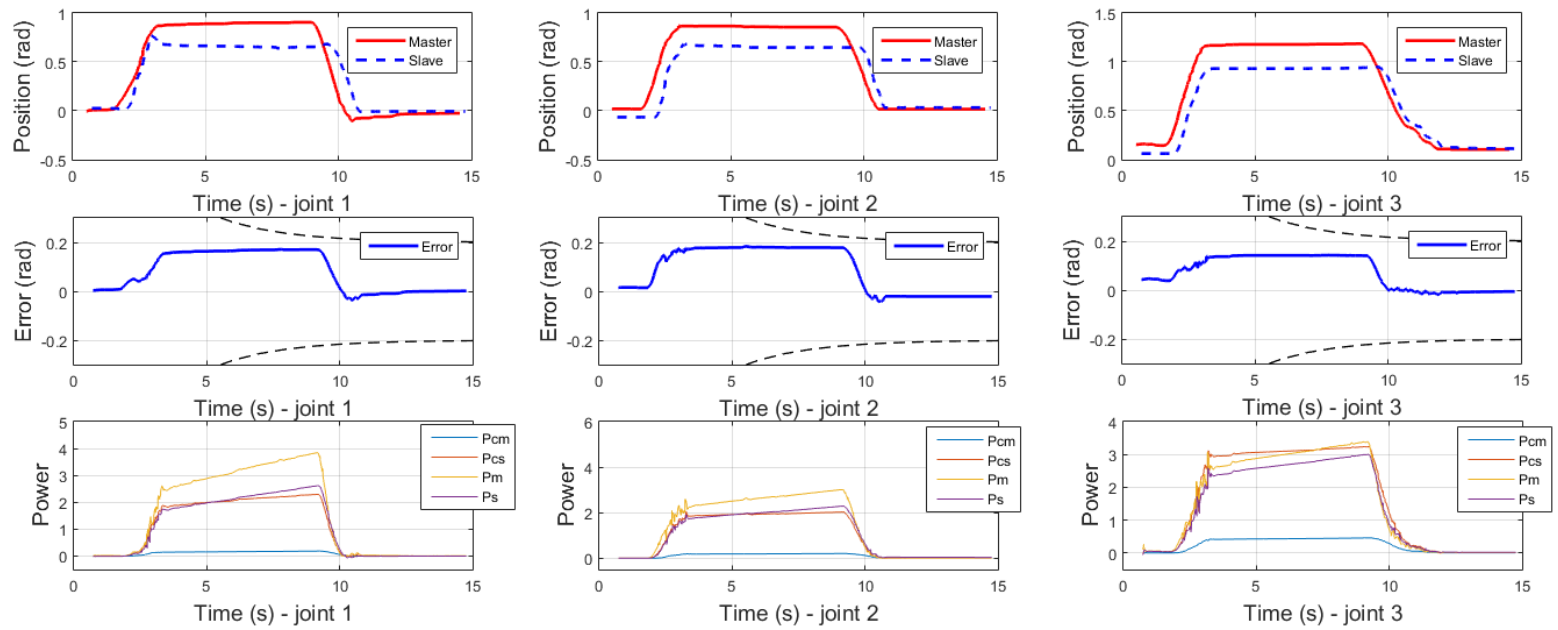

Fig.9. Position and observed power of our system during hard contact (slowly-varying delays) 

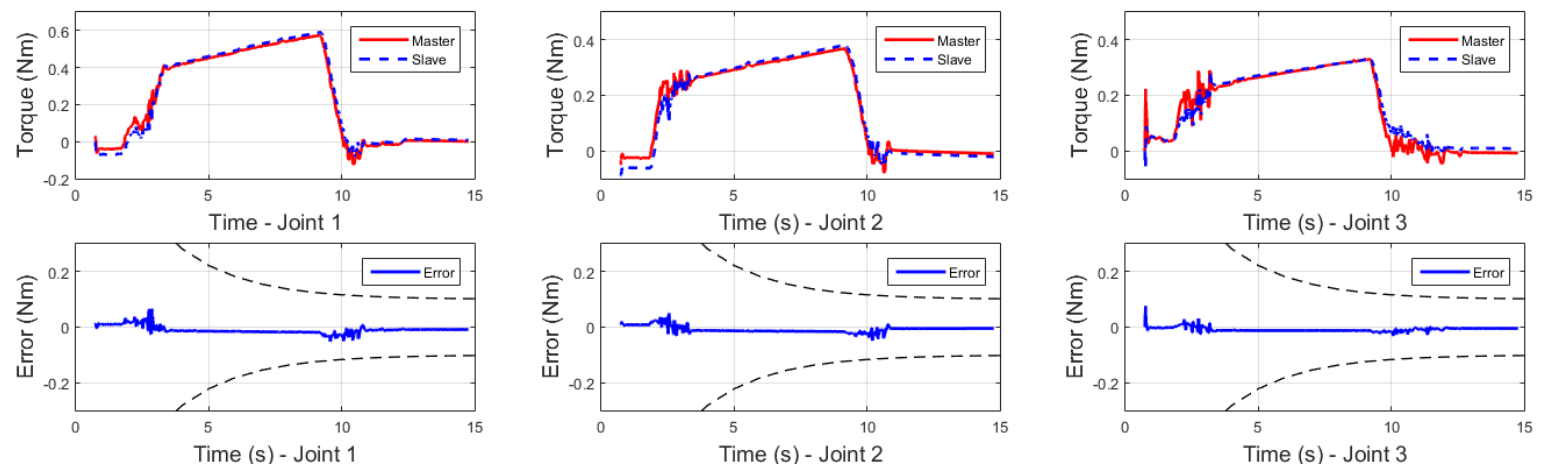

Fig.10. Torque of our system during hard contact (slowly-varying delays)
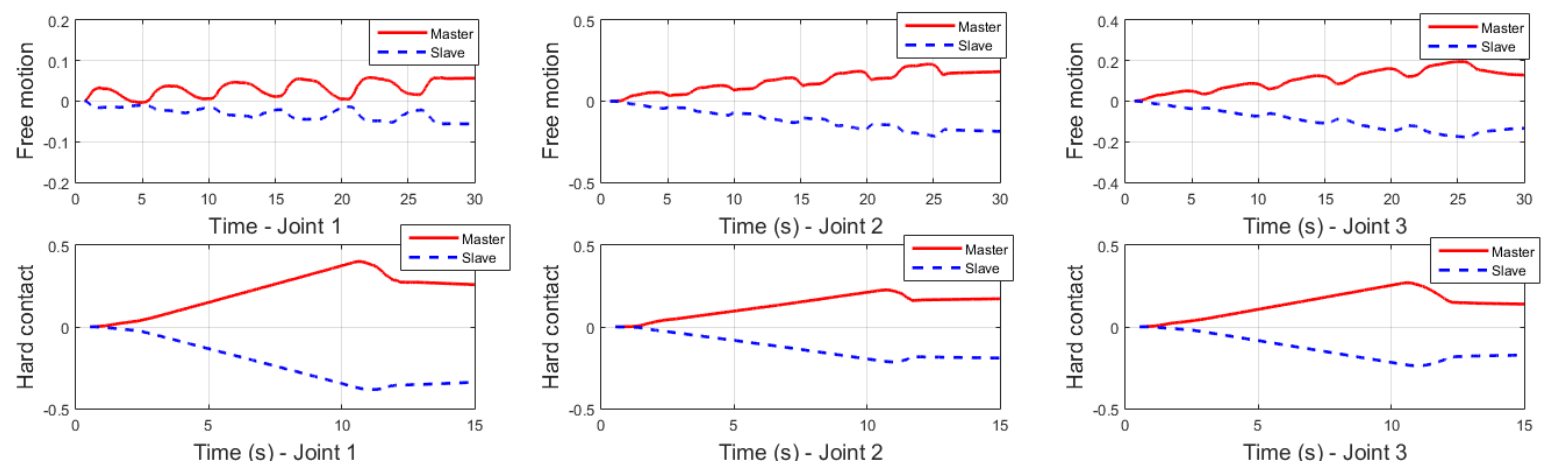

Fig.11. Output of FL controllers (slowly-varying delays)

\subsection{Sharply-varying delays}

In this section, the system's performance in the presence of large time delays with large variations are demonstrated. The values of time delays are around $2 \mathrm{~s}$. The rate of time delays is around 1. The proposed system is compared with our previous system in [20]. Fig.12 shows the position, torque and the related tracking errors of the system in [20] during free motion. In the presence of sharply-varying delays, the passivity controllers are launched in order to guarantee the system stability. Therefore, the position and torque tracking of the system in [20] are seriously degraded by the passivity controllers in [20] and large errors occur as shown in Fig.12. The operator feeling is dampen by the large feedback forces during free motion and the master can hardly drive the slave to move to the designated spot.

Figs.13 and 14 show the position, observed power signals and torque of the proposed system. Under sharply-varying delays, the proposed passivity controllers are launched by the proposed observers to guarantee the passivity of the master side, communication channels and the slave side. Owing to the proposed PPC, even under sharply-varying delays, highly accurate position and torque tracking is still derived and the errors are suppressed to be less than 0.2 .

Then, the slave robots in the two systems are driven by the operator to contact to the solid wall. Fig. 15 shows the positon, torque and the related tracking errors of the system in [20]. The environmental torque is nearly half of the human force, which means the system in [20] cannot efficiently conduct hard contact under sharply-varying delays. The position errors are also very large at the steady state $(2 s-9 s)$ as shown in Fig. 15 .

Figs. 16 and 17 show the position, observed power signals and torque of the proposed system during hard contact. As shown in Fig.16, Due to the large rate of time delays, large values of the control torque and position signals should have been reduced by the passivity controllers in both of the systems. However, with the designed PPC algorithm, the errors between the human torque and the environmental torque are actually suppressed under a preset value. So in the sharply-varying delays, the tracking errors of position and torque are much lower than those of the system in [20]. The signal perturbations in Fig.16 is caused by the large rate of time-varying delays. The proposed PPC efficiently suppress the errors between the human torque and the environmental torque and near-accurate torque tracking is still achieved during sharply-varying delays. The slight signal perturbations in Fig.16 is caused by the time-varying delays. The torque tracking errors are less than 0.2 on average. The steady-state position errors of the proposed system is also smaller than those of the system in [20] (less than 0.2). The output of the FL controllers are shown in Fig. 18.

We can conclude that by adding extra passivity observers and controllers, the proposed system can robustly guarantee the overall system's passivity (the passivity of the master, the communication channels and the slave). Compared with our previous system in [20], the proposed PPC is capable of restricting position and torque tracking errors in the presence of sharply-varying delays. Therefore, the proposed system can have better motion synchronization. 

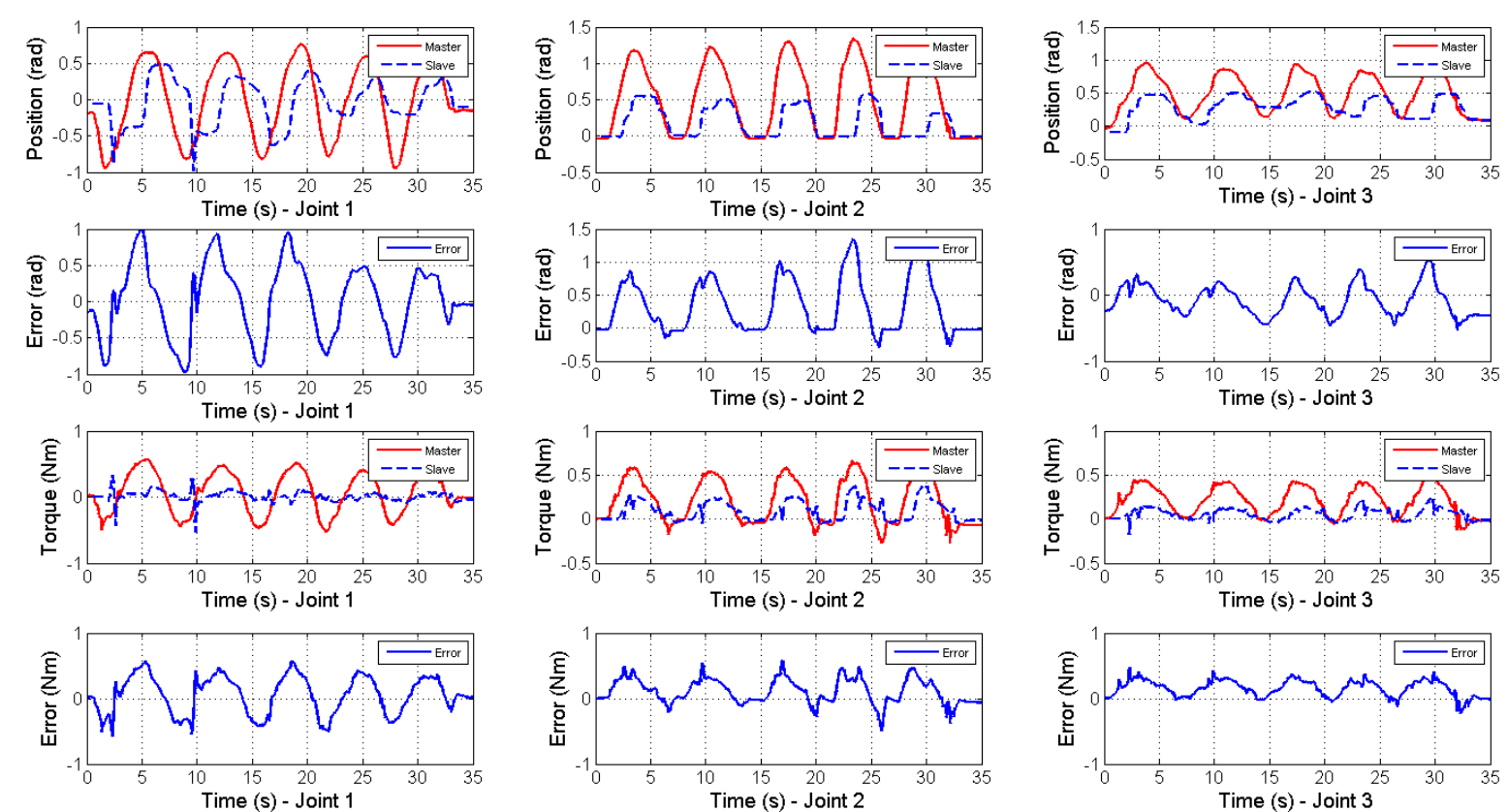

Fig.12. Position and torque tracking of the system in [20] during free motion (sharply-varying delays)
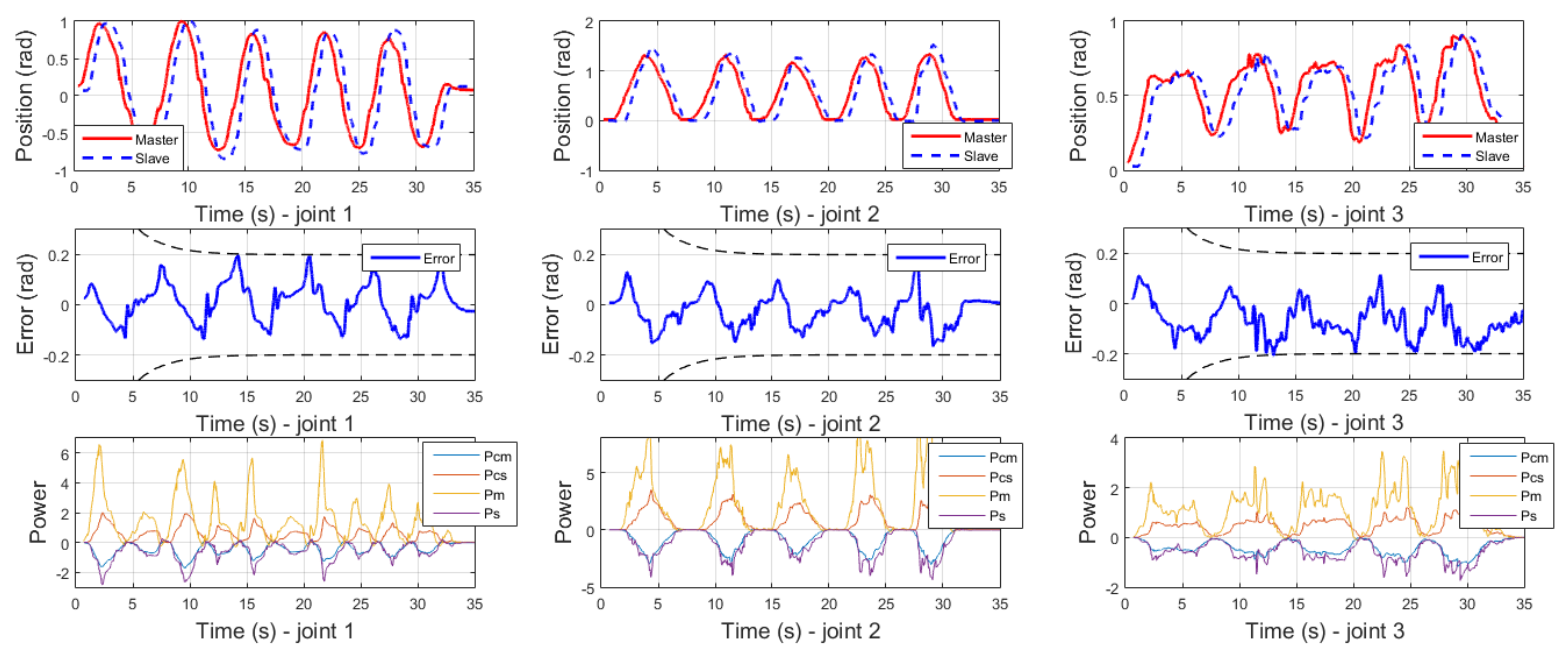

Fig.13. Position and observed power of our system during free motion (sharply-varying delays)
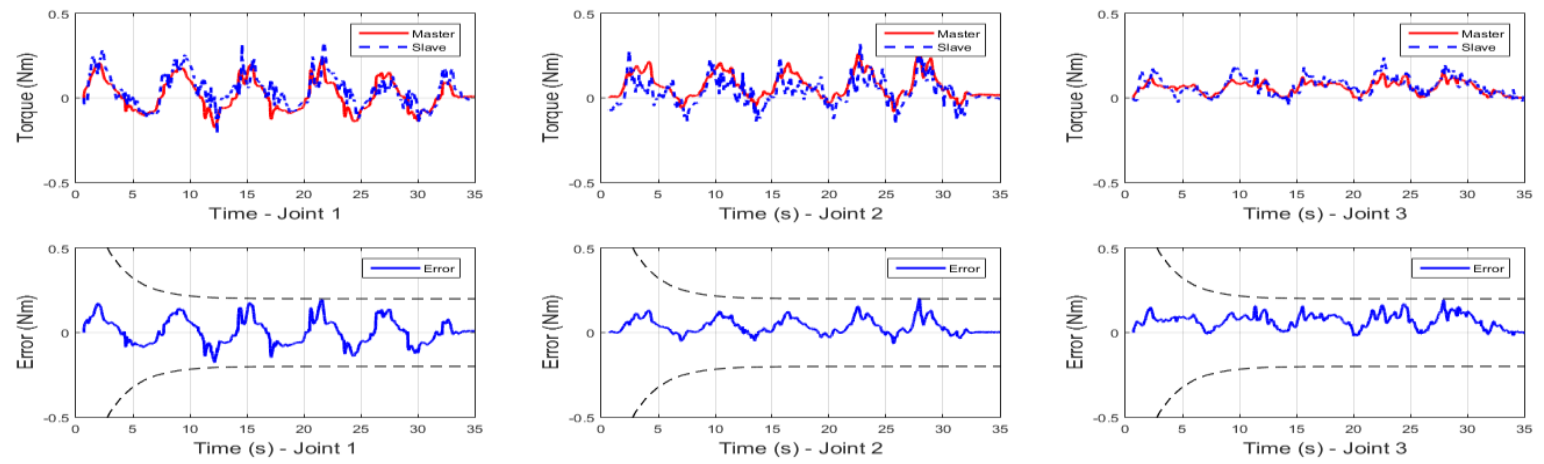

Fig.14. torque of our system during free motion (sharply-varying delays) 

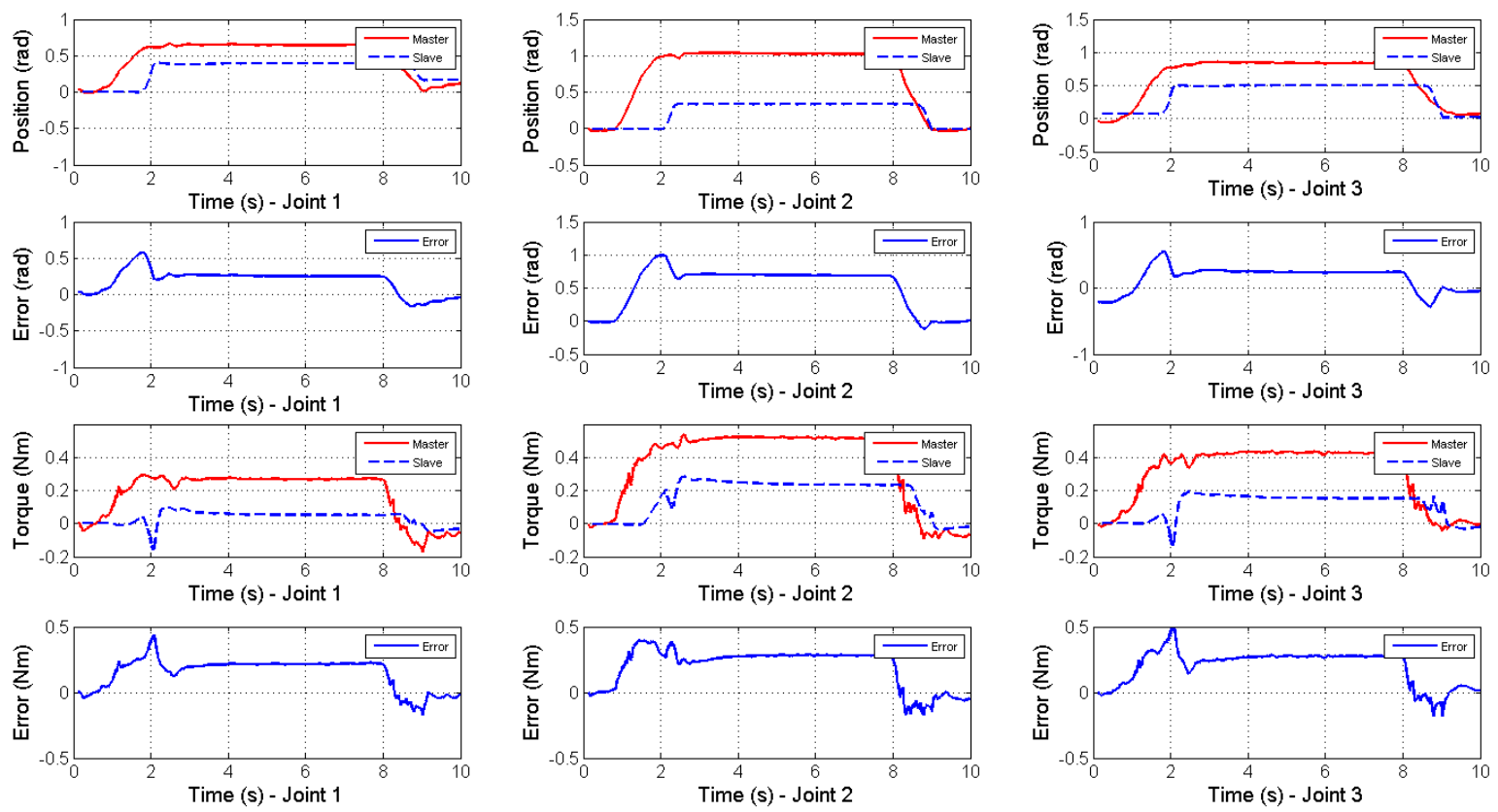

Fig.15. Position and torque tracking of the system in [20] during hard contact (sharply-varying delays)
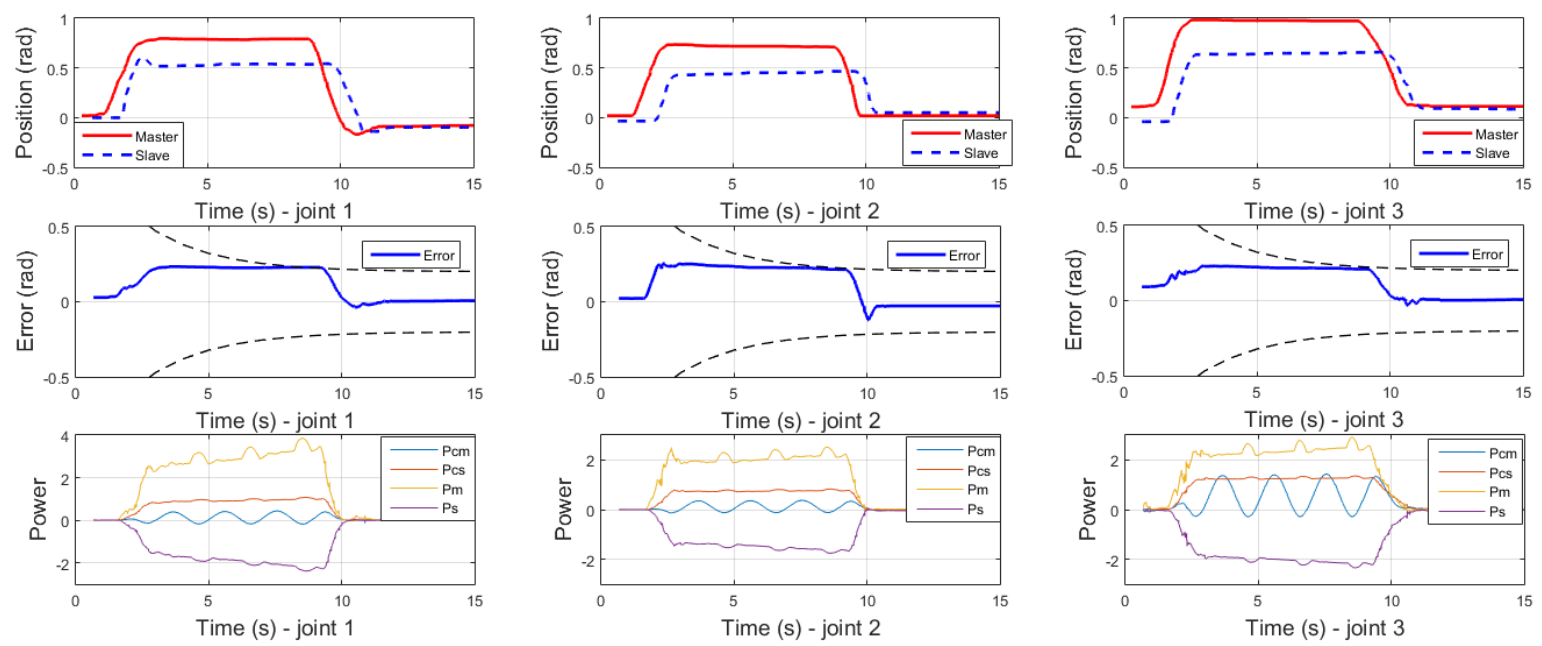

Fig.16. Position and observed power of our system during hard contact (sharply-varying delays)
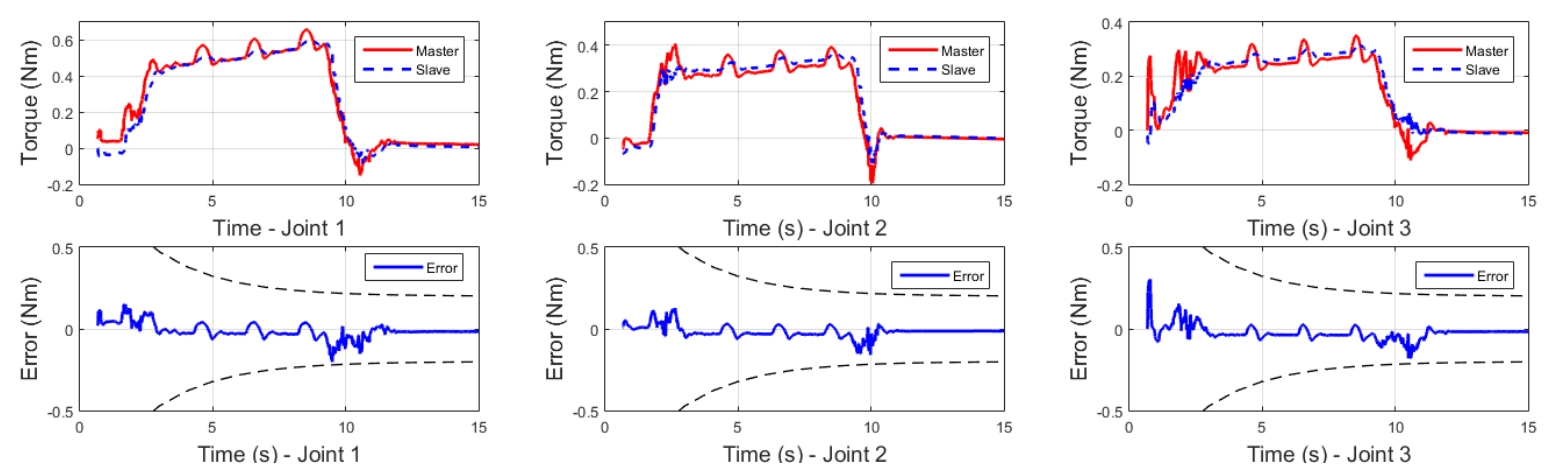

Fig.17. Torque of our system during hard contact (sharply-varying delays) 

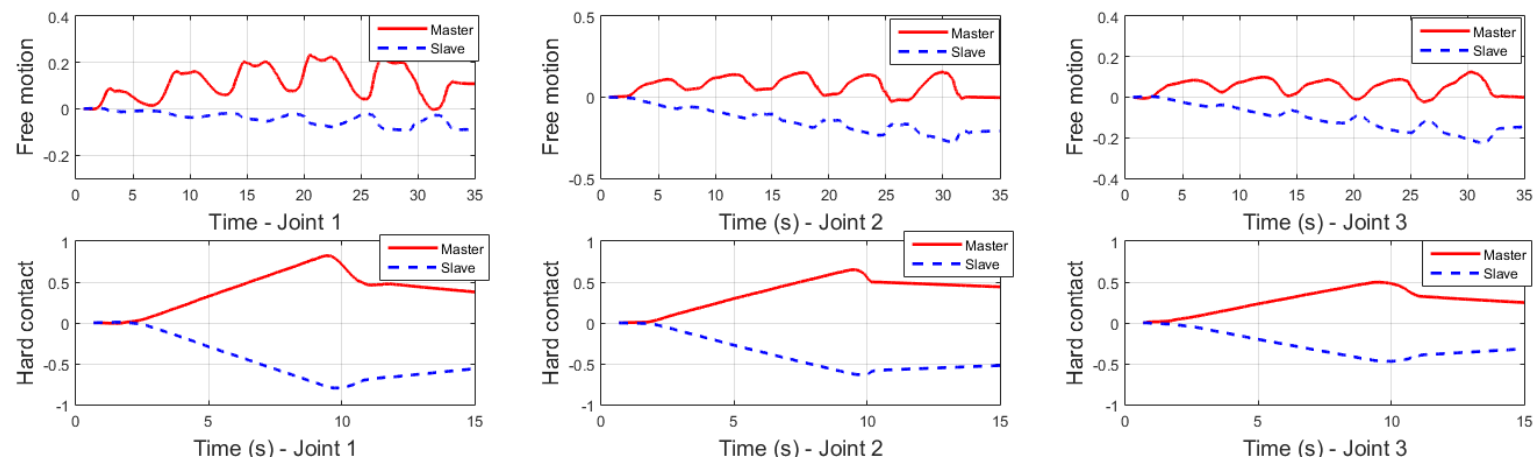

Fig.18. Output of FL controllers (sharply-varying delays)

\section{Conclusion}

In this paper, the wave-based TDPA is extended to guarantee the whole master-slave system's passivity by introducing extra passivity observer and controllers on the master and the slave sides. Furthermore, the prescribed performance control algorithm is extended to restrict the synchronization errors of the position and the velocity of the master and the slave and the measured human and environmental torques. By combining the extended TDPA and PPC algorithms, the passivity of the whole system is guaranteed and the system transparency is also simultaneously enhance to the extent that the slave can reasonably track the master's motion and the human operator can accurately feel the remote environment. Lyapunov functions are applied to analyze the system stability and the system's performance during diverse scenarios are validated through experimental work.

\section{Reference:}

[1] R. Kelly, A tuning procedure for stable PID control of robot manipulators, Robotica, 13(2): 141-148, 1995.

[2] A. Ilchmann, E. P. Ryan and S. Trenn, Tracking control: performance funnels and prescribed transient behavior, System control letter, 54(7): 655-670, 2005.

[3] C. P. Bechlioulis and G. A. Rovithakis, Adaptive control with guaranteed transient and steady state tracking error bounds for strict feedback systems, Automatica, 45(2): 532-538, 2009.

[4] X. L. Xie, Z. G. Hou, L. Cheng, C. Ji, M. Tan and H. Yu, Adaptive neural network tracking control of robot manipulators with prescribed performance, Proceedings of the Institution of Mechanical Engineers, Part I: Journal of Systems and Control Engineering, 225(6): 790-797, 2011.

[5] C. P. Bechlioulis, Z. Doulgeri and G. A. Rovithakis, Neuroadaptive force/position control with prescribed performance and guaranteed contact maintenance, IEEE Trans. Neur. Netw., 21(12): 1857-1868, 2010.

[6] Y. Karayiannidis and Z. Doulgeri, Model-free robot position regulation and tracking with prescribed performance guarantees, Robotics and Autonomous systems, 60(2): 214-226, 2012.

[7] A. K. Kostarigka, Z. Doulgeri and G. A. Rovithakis, Prescribed performance tracking for flexible joint robots with unknown dynamics and variable elasticity, Automatica, 49(5): 1137-1147, 2013.

[8] Yana, Yang, Hua Changchun, and Guan Xinping. "Adaptive prescribed performance control for nonlinear networked teleoperation system under time delay." Control Conference (CCC), 2014 33rd Chinese. IEEE, 2014.

[9] Yang, Yana, et al. "Adaptive neural network based prescribed performance control for teleoperation system under input saturation."Journal of the Franklin Institute352.5 (2015): 1850-1866.

[10] Yang, Yana, Changchun Hua, and Xinping Guan. "Synchronization control for bilateral teleoperation system with prescribed performance under asymmetric time delay." Nonlinear Dynamics (2015): 1-13.

[11] E. Nuno, R. Ortega, N. Barbanov and L. Basanez, A globally stable PD controller for bilateral teleoperators, IEEE Trans. on Robot., 24(3): 753-758, 2008.

[12] D. Lee and M. W. Spong, Passive bilateral teleoperation with constant time delay, IEEE Trans. on Robot., 22(2): 269-281, 2006.

[13] C. C. Hua and X. P. Liu, Delay-dependent stability criteria of teleoperation systems with asymmetric time-varying delays, IEEE Trans. on Robot., 26(5): 925-932, 2010.

[14] F. Hashemzadeh, I. Hassanzadeh and M. Tavakoli, Teleoperation in the presence of varying time delays and sandwich linearity in actuators, Automatica, 49(9): 2813-2821, 2013.

[15] C. Hua and Y. Yang, Bilateral Teleoperation Design With/Without Gravity Measurement, IEEE Trans. on Instru. And Measu., 61(12): 3136-3146, 2012.

[16] Nuño, E., Basañez, L., Ortega, R., \& Spong, M. W. (2009). Position tracking for non-linear teleoperators with variable time delay. The International Journal of Robotics Research, 28(7), 895-910.

[17] Nuño, Emmanuel, Luis Basañez, and Romeo Ortega. "Passivity-based control for bilateral teleoperation: A tutorial." Automatica 47.3 (2011): 485495.

[18] Rebelo, Joao, and Andre Schiele. "Time domain passivity controller for 4channel time-delay bilateral teleoperation." Haptics, IEEE Transactions on8.1 (2015): 79-89.

[19] Li, Hongbing, and Kenji Kawashima. "Bilateral teleoperation with delayed force feedback using time domain passivity controller." Robotics and Computer-Integrated Manufacturing (2015).

[20] Sun, Da, Fazel Naghdy, and Haiping Du. "Wave-variable-based Passivity Control of Four-channel Nonlinear Bilateral Teleoperation System under Time Delays." Mechatronics, IEEE Trans. on, online first.

[21] C. Passenbarg, A. Peer and M. Buss, "A survey of environment-, operatorand task-adapted controllers for teleoperation systems," Mechatronics 20, 787-801 (Oct. 2010).

[22] Chopra, Nikhil, Mark W. Spong, and Rogelio Lozano. "Synchronization of bilateral teleoperators with time delay." Automatica 44.8 (2008): 21422148.

[23] R. Anderson and M. Spong, "Bilateral control of teleoperators with time delay," IEEE Trans. Autom. Control, vol. 34, no. 5, pp. 494-501, May. 1989.

[24] R. J.Anderson andM.W. Spong, "Asymptotic stability for force reflecting teleoperators with time delay," Int. J. Robot. Res., vol. 11, no. 2, pp. 135$149,1992$.

[25] Chopra, Nikhil, Mark W. Spong, and Rogelio Lozano. "Synchronization of bilateral teleoperators with time delay." Automatica 44.8 (2008): 2142-2148.

[26] J. Ryu, D. Kwon, and B. Hannaford, "Stable teleoperation with timedomain passivity control," IEEE Trans. Robot. Autom., vol. 20, no. 2, pp. 365-373, Apr. 2004.

[27] J., Artigas, J. H., Ryu, C., Preusche, (2010). Time domain passivity control for position-position teleoperation architectures. Presence: Teleoperators and Virtual Environments, 19(5), 482-497.

[28] Y. Ye, Y.-J. Pan, and T. Hilliard, "Bilateral teleoperation with time-varying delay: A communication channel passification approach," IEEE/ASME Trans. Mechatronics, vol. 18, no. 4, pp. 1431-1434, Aug. 2013.

[29] Chawda, V.; OMalley, M.K., "Position Synchronization in Bilateral Teleoperation Under Time-Varying Communication Delays," Mechatronics IEEE/ASME Transactions on, vol.20, no.1, pp.245,253, Feb. 2015.

[30] Ryu, Jee-Hwan, Jordi Artigas, and Carsten Preusche. "A passive bilateral control scheme for a teleoperator with time-varying communication delay."Mechatronics 20.7 (2010): 812-823.

[31] Li, Hongbing, Kotaro Tadano, and Kenji Kawashima. "Model-based passive bilateral teleoperation with time delay." Transactions of the Institute of Measurement and Control (2014): 0142331214530423. 